\title{
ARTICLE
}

Received 18 Aug 2016 | Accepted 30 Mar 2017 | Published 8 Jun $2017 \quad$ DOl: 10.1038/ncomms15456

\section{Loss of Asxl2 leads to myeloid malignancies in mice}

\author{
Jianping $\mathrm{Li}^{1, \star}$, Fuhong $\mathrm{He}^{2, \star}$, Peng Zhang ${ }^{1, \star}$, Shi Chen ${ }^{1}$, Hui Shi ${ }^{1,3}$, Yanling Sun ${ }^{2,4}$, Ying Guo ${ }^{1}$, Hui Yang ${ }^{1}$, Na Man ${ }^{1}$, \\ Sarah Greenblatt ${ }^{1}$, Zhaomin Li ${ }^{1}$, Zhengyu Guo ${ }^{5}$, Yuan Zhou ${ }^{3}$, Lan Wang ${ }^{1}$, Lluis Morey ${ }^{1}$, Sion Williams ${ }^{1}$, Xi Chen ${ }^{1,6}$, \\ Qun-Tian Wang ${ }^{7}$, Stephen D. Nimer ${ }^{1}$, Peng $\mathrm{Yu}^{5}$, Qian-Fei Wang ${ }^{2,4}$, Mingjiang $\mathrm{Xu}^{1}$ \& Feng-Chun Yang ${ }^{1}$
}

ASXL2 is frequently mutated in acute myeloid leukaemia patients with $t(8 ; 21)$. However, the roles of $A S X L 2$ in normal haematopoiesis and the pathogenesis of myeloid malignancies remain unknown. Here we show that deletion of $A s \times 12$ in mice leads to the development of myelodysplastic syndrome (MDS)-like disease. Asx/2-/- mice have an increased bone marrow (BM) long-term haematopoietic stem cells (HSCs) and granulocyte-macrophage progenitors compared with wild-type controls. Recipients transplanted with $A s \times 12^{-/-}$and Asxl2 $2^{+/}$BM cells have shortened lifespan due to the development of MDS-like disease or myeloid leukaemia. Paired daughter cell assays demonstrate that Asx/2 loss enhances the self-renewal of HSCs. Deletion of Asx/2 alters the expression of genes critical for HSC self-renewal, differentiation and apoptosis in $\mathrm{Lin}^{-}{ }^{-} \mathrm{CKit}{ }^{+}$cells. The altered gene expression is associated with dysregulated $\mathrm{H} 3 \mathrm{~K} 27 \mathrm{ac}$ and $\mathrm{H} 3 \mathrm{~K} 4 \mathrm{me} 1 / 2$. Our study demonstrates that ASXL2 functions as a tumour suppressor to maintain normal HSC function.

\footnotetext{
${ }^{1}$ Sylvester Comprehensive Cancer Center, Department of Biochemistry and Molecular Biology, University of Miami Miller School of Medicine, 1011 NW 15 th Street, Room 417, Miami, Florida 33136, USA. ${ }^{2}$ Key Laboratory of Genomic and Precision Medicine, Collaborative Innovation Center of Genetics and Development, Beijing Institute of Genomics, Chinese Academy of Sciences, Beijing 100101, China. ${ }^{3}$ State Key Laboratory of Experimental Hematology, Institute of Hematology and Blood Diseases Hospital, Chinese Academy of Medical Sciences and Peking Union Medical College, Tianjin 300020, China. ${ }^{4}$ University of Chinese Academy of Sciences, Beijing 100049, China. ${ }^{5}$ Department of Electrical and Computer Engineering, and TEES-AgriLife Center for Bioinformatics and Genomic Systems Engineering, Texas A\&M University, College Station, Texas 77843, USA. ${ }^{6}$ Department of Public Health Sciences, University of Miami Miller School of Medicine, Miami, Florida 33136, USA. ${ }^{7}$ Department of Biological Sciences, University of Illinois at Chicago, Chicago, Illinois 60607, USA. * These authors contributed equally to this work. Correspondence and requests for materials should be addressed to Q.-F.W. (email: wangqf@big.ac.cn) or to M.X. (email: mxx51@miami.edu) or to F.-C.Y. (email: fxy37@med.miami.edu).
} 
aematopoietic stem cells (HSCs) have the ability to self-renew and give rise to all lineages of blood cells. The balance between HSC self-renewal and lineage specification is governed by the interplay between external and internal stimuli. Epigenetic factors have been shown to function as regulators for haematopoiesis. Epigenetic alterations are frequently associated with myeloid malignancies by establishing specific gene expression profiles ${ }^{1}$. Polycomb group (PcG) proteins are prominent epigenetic regulators and form multi-subunit complexes, which modify chromatin to maintain or repress the expression of their target genes ${ }^{2}$. ASXL1, 2 and 3 that comprise the additional sex comb-like family are putative PcG proteins, which share a common domain architecture of conserved aminoterminal ASXN, ASXH domains and a carboxy-terminal plant homeodomain (plant homeodomain finger) ${ }^{3}$. As a chromatin regulator, ASXL1 has been shown to exert its function by recruiting the polycomb repressive complex 2 , which adds methyl mark(s) on histone H3 lysine 27 (H3K27me1, H3K27me2 and $\mathrm{H} 3 \mathrm{~K} 27 \mathrm{me} 3)^{4-6}$. Asxl1 loss leads to the development of myeloid malignancies in mice, which is associated with dysregulation of H3K27me3 (refs 6,7). Lai et al. ${ }^{8}$ previously reported that ASXL2 regulates gene expression in the heart at cell-type and stagespecific manners. Enhancers and other distal regulatory elements are critical for context-specific gene regulation. Such elements are associated with characteristic chromatin marks, including $\mathrm{H} 3 \mathrm{~K} 4 \mathrm{me} 1$ and H3K27ac, which facilitate their identification.

Somatic mutations and chromosomal translocations are major drivers of a range of haematopoietic malignancies. Genomic studies have revealed that $A S X L 1$ and $A S X L 2$ are frequently mutated in myeloid malignancies ${ }^{9-11}$. ASXL1 mutations occur in multiple spectra of myeloid malignancies ${ }^{9,12}$. In contrast, ASXL2 mutations are largely restricted to patients with $t(8 ; 21)$ acute myeloid leukaemia (AML), both in children and in adults at an overall incidence of $\sim 23 \%$ (refs 13,14). Interestingly, ASXL1 and ASXL2 mutations are mutually exclusive in $t(8 ; 21)$ AML patients ${ }^{13}$, suggesting a common mechanism in promoting myeloid malignancies. Despite intense investigation, the role of ASXL2 in normal and malignant haematopoiesis remains unknown.

In the current study, utilizing a murine model of Asxl2 loss, we sought to explore the role of ASXL2 in normal and malignant haematopoiesis, and to identify the mechanisms by which Asxl2 loss contribute to myeloid malignancies. We showed that Asxl2 loss led to a myelodysplastic syndrome (MDS)-like disease in mice as evidenced by pancytopenia, dysplastic features of myeloid cells and skewed differentiation towards myeloid lineages. Asxl2 ${ }^{-/-}$mice had increased frequency of long-term (LT) HSCs. Transplantation of bone marrow (BM) cells from $A s x l 2^{-/-}$or $A s x l 2^{+/-}$mice led to a leukaemic transformation in the lethally irradiated recipients, indicating a cell-autonomous effect of $A s x l 2$ loss on the pathogenesis of myeloid malignancies. Convergent analyses of RNA sequencing (RNA-seq) and chromatin immunoprecipitation (ChIP) assays followed by sequencing (ChIP-seq) data in $\mathrm{BM} \mathrm{Lin}{ }^{-} \mathrm{cKit}^{+}$(LK) cells identified a subset of differentially expressed genes (DEGs), enriched with critical genes for HSC function and apoptosis, as well as myelopoiesis. Importantly, the altered gene expression was associated with dysregulated histone marks, including $\mathrm{H} 3 \mathrm{~K} 27 \mathrm{ac}$ and $\mathrm{H} 3 \mathrm{~K} 4 \mathrm{me} 1 / 2$. Our results demonstrate a critical role of ASXL2 in the maintenance of normal HSC functions.

\section{Results}

Asxl2 is required for normal haematopoiesis. To determine Asxl2 expression in wild-type (WT) haematopoietic lineages, different haematopoietic cell subpopulations were used for quantitative real-time PCR (qPCR). Asxl2 was ubiquitously expressed in all cell subpopulations/lineages examined, including $\mathrm{Lin}^{-} \mathrm{Sca}{ }^{+}$ $\mathrm{cKit}^{+}$(LSK), common myeloid progenitor, granulocyte-monocyte progenitor (GMP), megakaryocyte-erythrocyte progenitor, neutrophils (NEs), monocytes, erythrocytes (E), megakaryocytes, $\mathrm{B}$ cells and T cells (Supplementary Fig. 1a).

To elucidate the effects of Asxl2 on haematopoiesis, we performed a series of haematopoietic phenotypic analyses using 6-8-week-old $A s x l 2^{+/-}$and Asxl2 ${ }^{-/-}$mice. The Asxl2deficient mice were generated previously by inserting a gene trap cassette into the first intron of $A s x l 2$, which disrupts ASXL2 expression ${ }^{15}$. Successful deletion of Asxl2 in haematopoietic cells was shown by PCR, qPCR and western blotting, respectively (Supplementary Fig. 1b-d). Of note, Asxl2 deletion did not affect the Asxl1 messenger RNA level in $A s x l 2^{-/-}$BM cells (Supplementary Fig. 1e).

Peripheral blood (PB) counts revealed pancytopenia in As $x 12^{-/-}$mice (Fig. 1a). However, the proportion of NEs was significantly higher in the $\mathrm{PB}$ of $A s \times l 2^{-/}-$mice compared with WT controls (Fig. 1a). May-Grünwald-Giemsa-stained PB smears of $A s \times l 2^{+9}$ and $A s \times l 2^{-1-}$ mice showed dysplastic features, including hyposegmented (bilobed) NEs with fine nuclear bridging (consistent with pseudo Pelger-Huët), asymmetric nuclear structure and increased number of polychromatophilic red blood cells and Howell-Jolly bodies in erythrocytes (Fig. 1b). Analyses of BM histologic sections of Asxl2 $2^{+/}$and $A s x l 2^{-1-}$ mice revealed an increase in the proportion of myeloid cells and relatively decreased erythroid islands compared with WT controls (Fig. 1c). Consistently, Asxl2 ${ }^{-/-}$mice had an increased myeloid/erythroid ratio compared with WT mice (WT: $0.92 \pm 0.21$ versus $A s x l 2^{-/-}$: $2.20 \pm 1.39)$. Dysplastic features were also frequently seen in As $x l 2^{+/-}$and $A s x l 2^{-/-}$BM sections, and cellular cytospins, including abnormal nuclear and cytoplasm proportion, nuclear budding and fragmentocytes (Fig. 1c). In addition, the BM cellularity in $A s x l 2^{-1}$ femur was significantly lower than that in WT controls (Supplementary Fig. 1f).

Despite the lower body weight, Asxl2 ${ }^{-/-}$mice exhibited splenomegaly with a higher cellularity compared with WT mice (Fig. 1d and Supplementary Fig. 1g). The histologic analysis of the Asxl2 $2^{+1-}$ and $A s x l 2^{-1-}$ spleen sections showed disrupted splenic architecture with an increased proportion of myeloid cells (Fig. 1e). These data suggest that ASXL2 is required for normal haematopoiesis and loss of Asxl2 in mice leads to haematological characteristics resembling an MDS-like disease.

ASXL2 is required for the maintenance of GMP and LT-HSCs. To further characterize the Asxl2-loss-mediated dysregulation of haematopoiesis, flow cytometric analyses were performed on $\mathrm{PB}, \mathrm{BM}$ and spleen cells of WT, $A s \times l 2^{+1-}$ and $A s \times l 2^{-1-}$ mice. An increased proportion of granulocytic/monocytic cells $\left(\mathrm{Gr}^{+} / \mathrm{Macl}^{+}\right)$was observed in the $\mathrm{PB}, \mathrm{BM}$ and spleens of Asxl2 ${ }^{-/-}$mice compared with WT mice (Fig. 2a,b and Supplementary Fig. 2a,b). Immunohistochemical staining showed massive accumulation of myeloperoxidase (MPO)-positive cells in the spleens of $A s x l 2^{+I^{-}}$and $A s x l 2^{-I-}$ mice compared with WT spleens (Supplementary Fig. 2c,d), verifying the increased proportion of myeloid cells in the Asxl2 $2^{+-}$and As $x l 2^{-1-}$ spleens. In contrast, a decreased proportion of the $\mathrm{CD} 71^{+} /$Ter119 ${ }^{+}$population was observed in the BM of Asxl2 ${ }^{-/-}$mice compared with WT controls (Supplementary Fig. 2e), suggesting a role of ASXL2 in erythropoiesis in vivo.

As the frequency and number of lymphocytes were significantly lower in the PB of $A s x l 2^{-1-}$ mice compared with WT mice, we next performed flow cytometric analysis to further determine whether Asxl2 deletion impairs lymphocyte development. Lower 
frequencies of LMPP (lymphoid-primed multipotent progenitor) and CLP (common lymphoid progenitor) were observed in the BM of $A s x l 2^{-1-}$ mice compared with WT and $A s x l 2^{+/-}$mice (Supplementary Fig. 3a). In addition, lower percentages of pre-B cells $\left(\mathrm{cKit}^{+} / \mathrm{B}^{2} 20^{+}\right.$and $\mathrm{CD}_{2} 5^{-} / \mathrm{IgM}^{+}$) were observed in Asxl2 ${ }^{-1-}$ BM cells (Supplementary Fig. 3b). A higher proportion of DN1 $\left(\mathrm{CD} 44^{+} / \mathrm{CD} 25^{-} / \mathrm{CD} 4^{-} / \mathrm{CD} 8^{-}\right)$and a lower percentage of $\mathrm{CD} 4^{+} / \mathrm{CD} 8{ }^{+}$cell population were observed in the thymus of
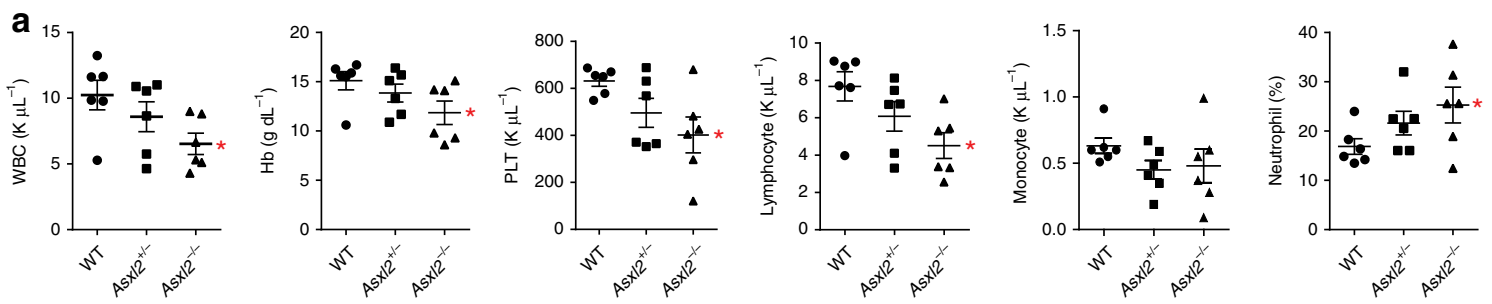

b
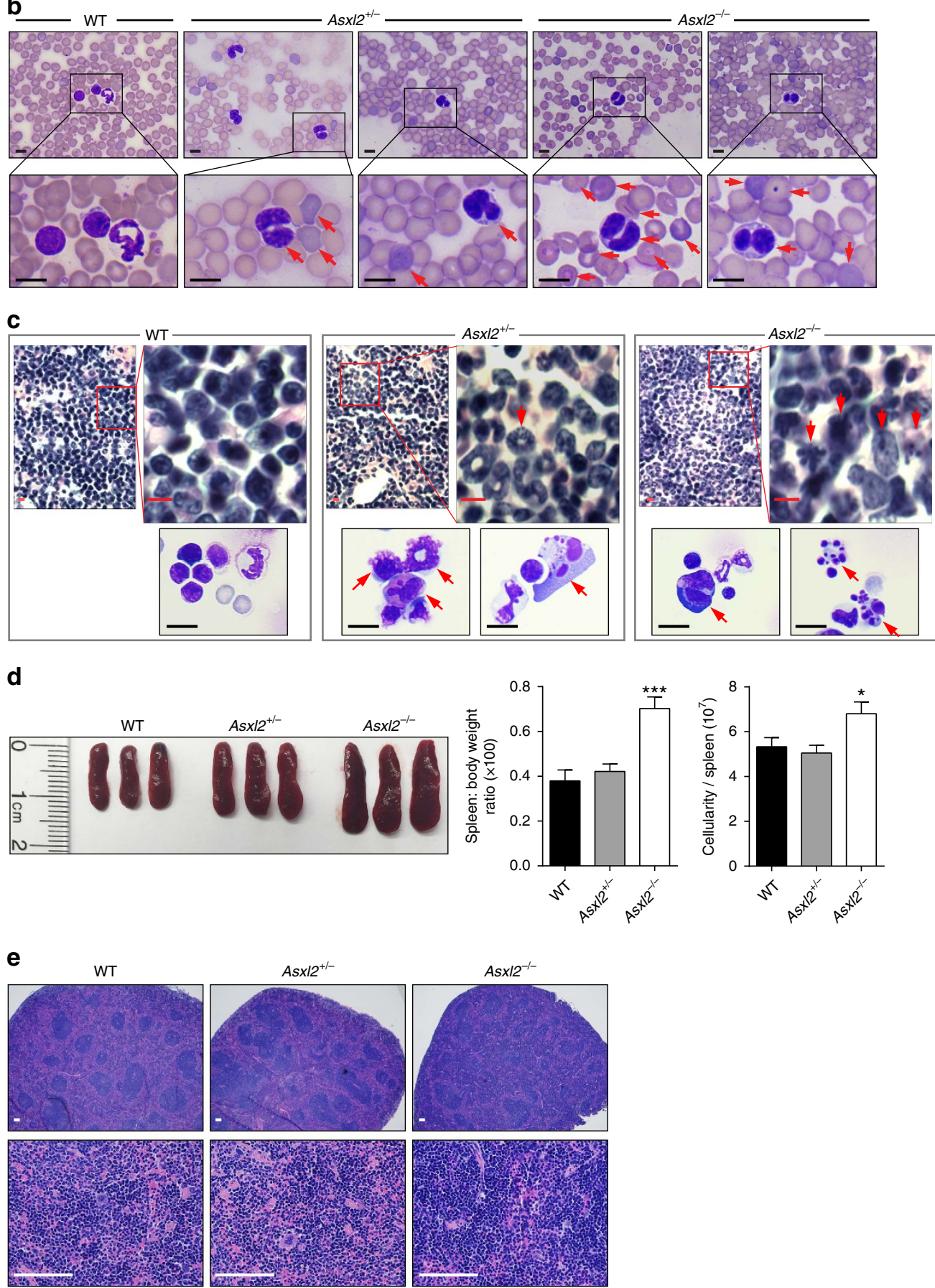
Asxl2 $2^{-1-}$ mice compared with WT and Asxl2 ${ }^{+/-}$mice (Supplementary Fig. 3c). These data suggest that deletion of Asxl2 impairs the development of lymphocytes.

To assess whether Asxl2 deletion affects the pool of haematopoietic stem/progenitor cells (HSC/HPCs), we performed phenotypic analysis on the BM cells of WT, Asxl2 $1-$ and Asxl2 $2^{-T}$ mice by flow cytometry. Asxl2 ${ }^{-/}$BM cells had a significantly higher proportion of LSK cells compared with WT $\mathrm{BM}$ cells (Fig. 2c,d). The $\mathrm{Lin}^{-} \mathrm{cKit}^{+} \mathrm{Scal}{ }^{-}\left(\mathrm{LKS}^{-}\right)$cell populations in the $\mathrm{BM}$ of $A s x l 2^{-/-}$mice were similar to that of WT mice (Supplementary Fig. 3d). Further analysis showed that deletion of Asxl2 significantly increased the LT-HSCs (LSKCD34 ${ }^{-}$FLK2 $^{-}$cells) and short-term-HSCs (LSKCD34 ${ }^{+}$ FLK2 ${ }^{-}$cells) in the BM compared with WT and Asxl2 ${ }^{+/-}$mice (Fig. 2e,f). The percentage of multipotential progenitor cells was similar in the BM of $A s x l 2^{-1-}$, WT and $A s x l 2^{+/-}$mice (Supplementary Fig. 3d). When each myeloid progenitor population was analysed within the $\mathrm{LKS}^{-}$populations, the percentage of GMP populations were significantly higher, whereas megakaryocyte-erythrocyte progenitor and common myeloid progenitor populations were slightly lower in the BM of $A s \times l 2^{-/-}$mice compared with WT and Asxl2 $2^{+/-}$mice (Fig. 2g,h and Supplementary Fig. 3d). Will et al. ${ }^{16}$ reported that higher-risk MDS have a higher proportion of GMP. The higher proportion of GMP in Asxl2-deficient mice may associate with disease progression in vivo.

Interestingly, aged $A s \times l 2^{-/-}$mice (around 12 months of age) had significantly higher white blood cell and NE counts in the PB than WT and Asxl2 ${ }^{+/-}$mice (Supplementary Fig. 4a). These aged $A s \times l 2^{-/-}$mice also exhibited weight loss, splenomegaly and pale femurs (Supplementary Fig. 4b). Flow cytometric analysis showed an increased proportion of $\mathrm{Grl}^{+} / \mathrm{Mac1}^{+}$cells (Supplementary Fig. 4c), higher percentage of LT-HSCs and GMPs (Supplementary Fig. 4d,e) in their BM.

Fetal liver (FL) is an important haematopoietic site in mice. Flow cytometric analysis on the FL cells of WT, Asxl2 ${ }^{+/-}$and Asxl2 ${ }^{-I}$ embryos (day 12.5) mice revealed increased multipotential progenitor cell and $\mathrm{Gr} 1^{+} / \mathrm{Mac}^{+}$cell populations in Asxl2 ${ }^{-1-}$ FL cells, whereas the frequencies of LSK and GMP were comparable among the three genotypes (Supplementary Fig. 5a). Consistently, the frequencies of colony-forming unit (CFU)- mixed colonies of granulocytes/macrophage (GM), E and megakaryocytic cells, CFU-GM and burst forming unit-E were significantly higher in $A s x l 2^{-1-}$ FL cells than in WT and As $x l 2^{+/-}$cells (Supplementary Fig. 5b).

Collectively, these data suggest that ASXL2 is required for the maintenance of normal HSC/HPC pools and lineage commitment in vivo.

Deletion of Asxl2 increases HSC self-renewal capacity. To determine the effect of Asxl2 loss on the function of HSC/HPCs, we next assayed the frequency of CFU cells (CFU-C) in the BM and spleens of WT, $A s x l 2^{+/-}$and $A s x l 2^{-/-}$mice. The frequencies of CFU-Cs, CFU-GM and CFU-mixed colonies of GM, E and megakaryocytic cells were significantly higher in the BM and spleens of $A s x l 2^{-/-}$mice (Fig. 3a and Supplementary Fig. 6a), suggesting a preferential myeloid progenitor increment. To determine the proliferative potential of multipotent progenitor cells, we assayed high proliferative potential colony-forming cells (HPP-CFC) using double-layer agar cultures $^{17}$. The frequencies of HPP-CFC were significantly higher in the $\mathrm{BM}$ of $A s x l 2^{+/-}$and $A s x l 2^{-1-}$ mice compared with WT mice (Fig. 3b).

Balanced symmetric and asymmetric cell division is required for the preservation of normal stem cell pool and production of blood cells. To further determine whether loss of Asxl2 alters cell fates of HSC/HPCs, paired daughter cell assays ${ }^{18}$ were performed to assess the proportions of symmetric self-renewal, symmetric division and asymmetric division using CD34- ${ }^{-}$LSK cells isolated from WT and Asxl2 ${ }^{-/-}$BM. Asxl2 loss resulted in a higher proportion of cells with symmetric self-renewal capacity (62\%) compared with WT cells (33\%, Fig. 3c). In contrast, the cells with symmetric differentiation potential were significantly reduced in Asxl2 ${ }^{-1-} \mathrm{CD}^{-} 4^{-}$LSK cells (18\%) compared with WT cells (40\%), whereas the frequency of cells that underwent asymmetric differentiation in Asxl2 $2^{-/-} \mathrm{CD} 34^{-}$LSK cells was comparable to that of WT cells (Fig. 3c). To determine the replating potential of CD34- LSK cells over time, we replated the cells that underwent symmetric self-renewal. Although a steady decline of replating potential was observed in WT cells, a significantly higher replating potential was observed over three successive replating times in $A s x l 2^{-1-}$ cells (Fig. 3d). In addition, the progeny of the single cell culture showed that $A s x l 2^{-/-}$CD $34^{-}$LSK cells preferentially gave rise to larger proportions of granulocytic/ monocytic cells, whereas the WT CD34- LSK cells had the potential to differentiate into diverse cell lineages, including erythroid cells and myeloid cells (Fig. 3e). These data indicate that ASXL2 is critical for maintaining the balance of symmetric and asymmetric divisions in HSCs.

The changes in the HSC/HPCs frequencies in vivo can be associated with altered cell proliferation and/or apoptosis. We then examined whether Asxl2 loss affected cell survival of HSC/HPCs by flow cytometric analysis on BM cells following Annexin $\mathrm{V}$ and 7-amino-actinomycin D (7-AAD) staining. As $x l 2^{-/-}$LK cells had a significantly lower proportion of Annexin $\mathrm{V}^{+}$cells than WT cells (Fig. 3f,g). In contrast, a higher percentage of Annexin $\mathrm{V}^{+}$cells was observed in matured cells $\left(\mathrm{Lin}^{+}\right)$of $A s \times l 2^{-/-}$BM than that of WT BM (Supplementary Fig. 6b,c). These data indicate that deletion of Asxl2 led to a reduced apoptosis of HSC/HPCs, but an increased apoptosis in more differentiated BM cells. Furthermore, 5-bromodeoxyuridine (BrdU) incorporation assays revealed an increased frequency of $A s x l 2^{+/-}$and $A s x l 2^{-/-}$LK cells at S-phase than WT LK cells, suggesting a higher proliferation of Asxl2 $2^{+/-}$and $A s x l 2^{-/-}$LK cells (Supplementary Fig. 6d). These results demonstrate that loss of $A s x l 2$ altered the apoptosis and proliferation of LK cells.

\footnotetext{
Figure 1 | Development of an MDS-like disease in Asx/2-/- mice. (a) Parameters of PB were summarized from WT, Asx/2+/- and Asx/2-/- mice at 6-8 weeks of age (six mice per genotype). (b) May-Grünwald-Giemsa stained PB smears from representative WT, Asx/2+/- and Asx/2-/- mice were shown. The PB smears of $A s \times 12^{+/-}$and $A s \times 12^{-1-}$ mice showed dysplastic features, including hyposegmented (bilobed) NEs with fine nuclear bridging consistent with pseudo Pelger-Huët, asymmetric nuclear structure and increased number of polychromatophilic red blood cells (RBCs) and Howell-Jolly bodies in erythrocytes. Scale bar, $10 \mu \mathrm{m}$. (c) Haematoxylin and eosin (H\&E) stained sections of femurs (upper panels) and May-Grünwald-Giemsa stained cytospins (lower panels) from representative WT, $A s \times 12^{+/-}$and $A s \times 12^{-/-}$mice (6-8 week old). Scale bar, $10 \mu \mathrm{m}$. (d) Enlarged spleens in Asx/2-/mice. Shown are representative photographs of spleens of three sets of WT, Asx/2+/- and Asx/2-/- mice (6-8 week old), spleen/body weight ratio $(\times 100)$ and spleen cellularity for each genotype of mice (six mice per genotype). (e) H\&E staining of paraffin-embedded sections of spleens from representative WT, Asx/2+/- and Asx/2- /- mice (6-8 week old). Red arrows indicate dysplastic cells. Scale bar, $100 \mu \mathrm{m}$. Data are presented as mean \pm s.e.m. Comparisons among the groups were performed by one-way analysis of variance. ${ }^{\star} P<0.05$ and ${ }^{\star \star \star} P<0.001$.
} 


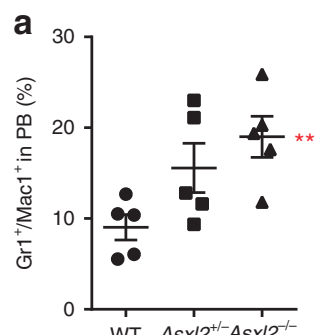

WT $A s \times 12^{+/-} A s \times 12^{-1-}$

$\mathbf{b}_{\mathrm{PB}}$

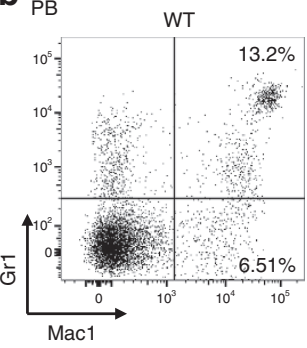

C Gated on Lin
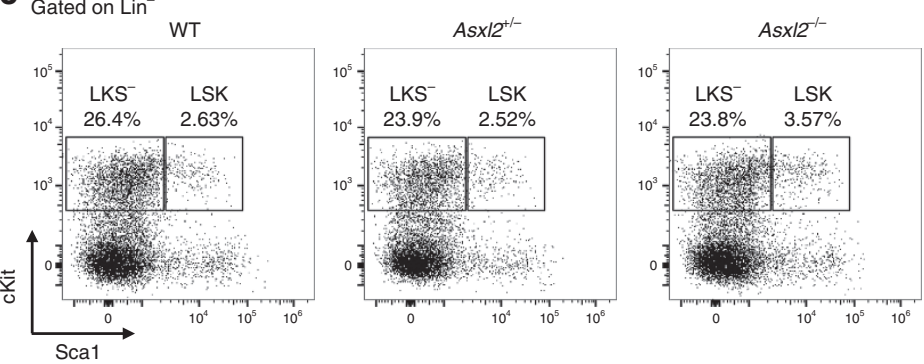

e Gated on LSK
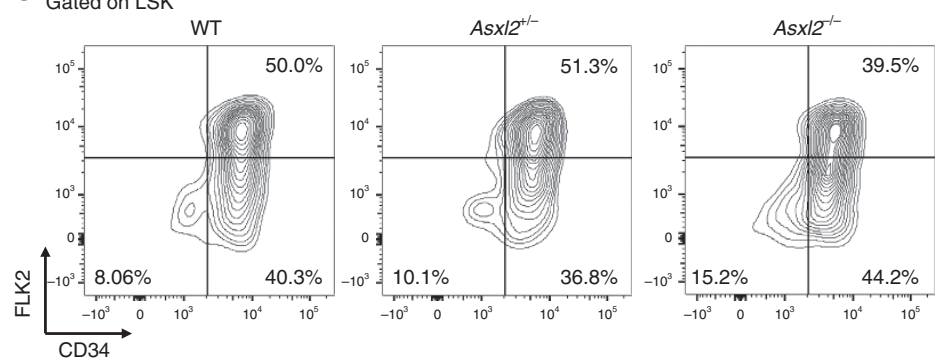

9 Gated on LKS
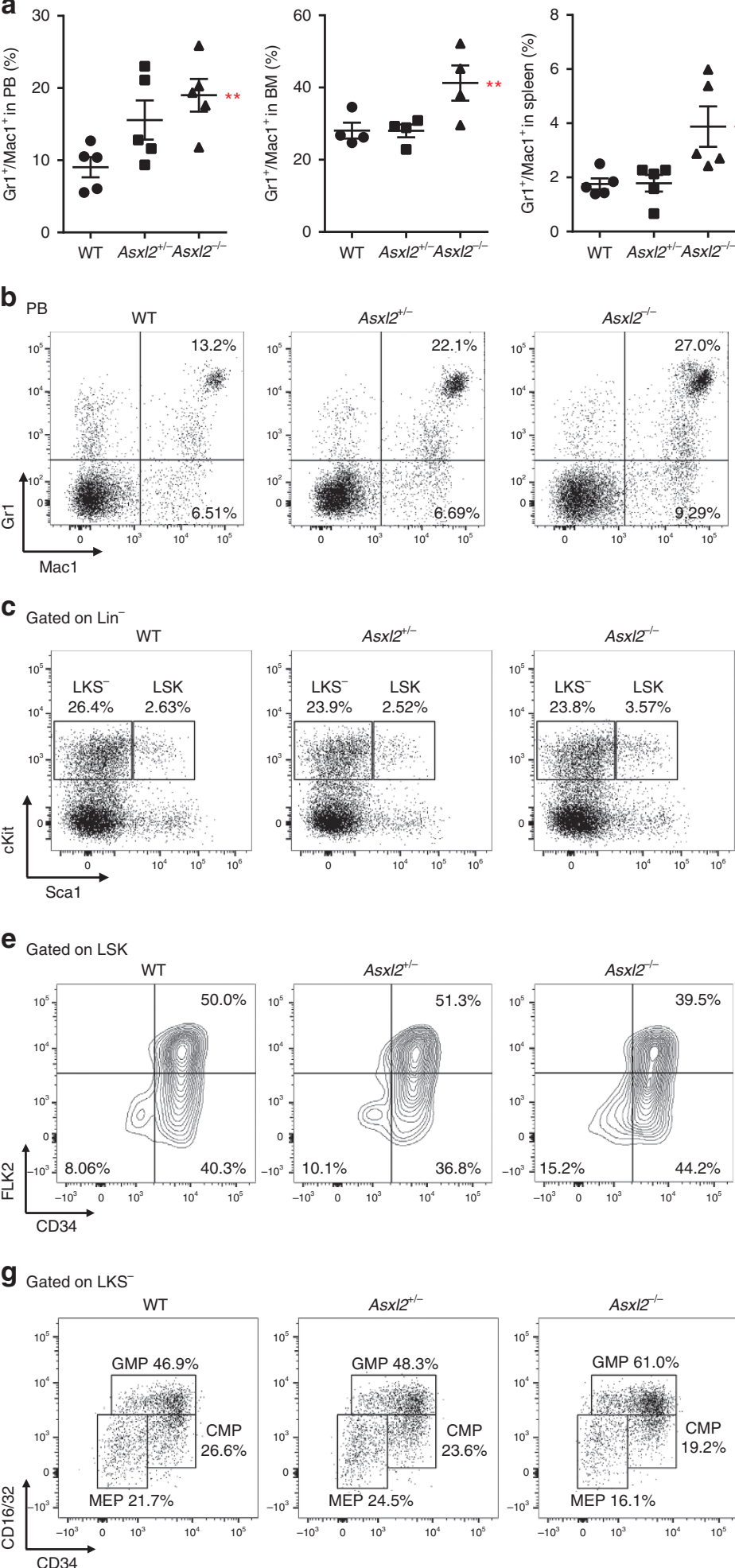
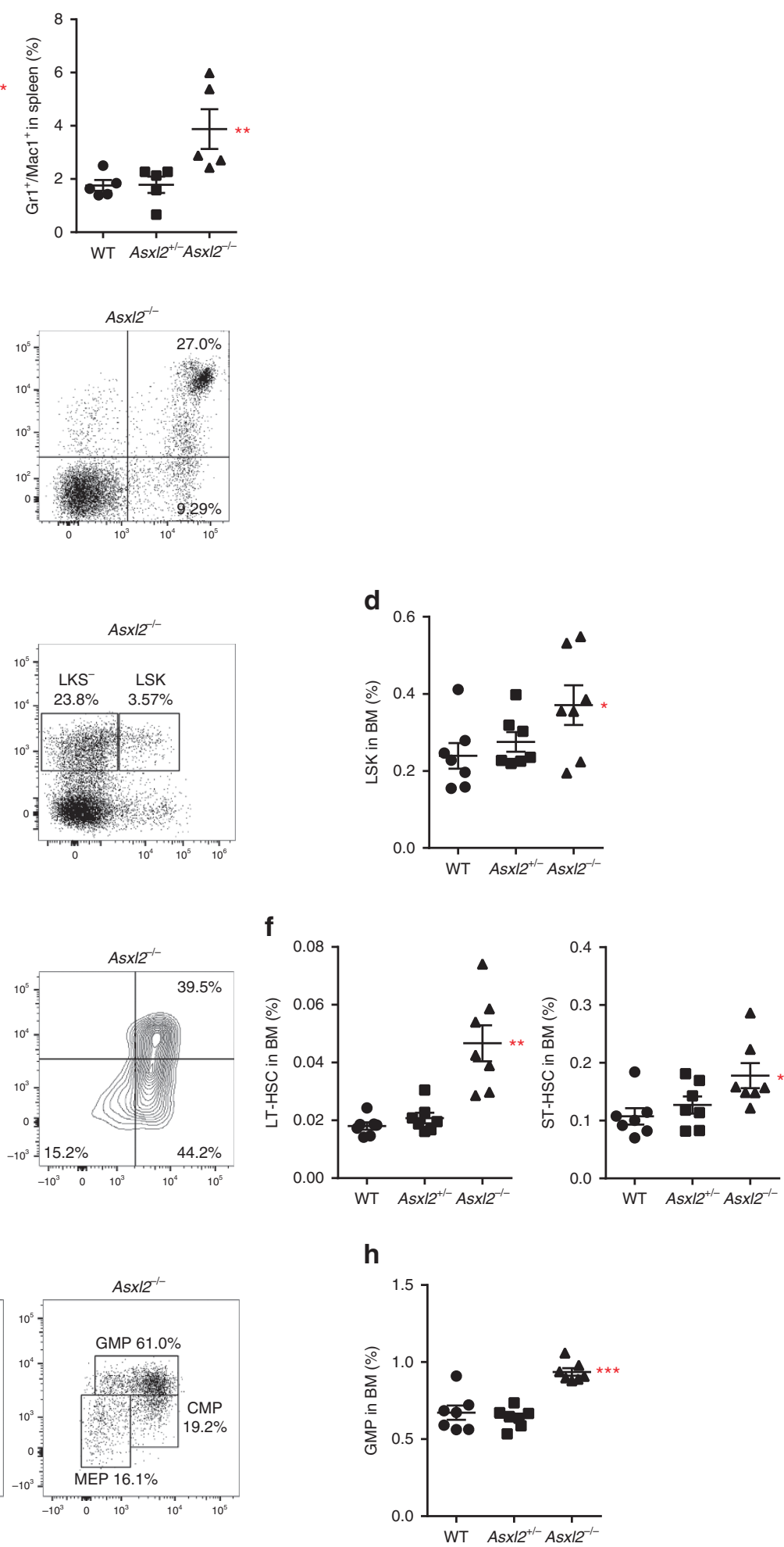

h

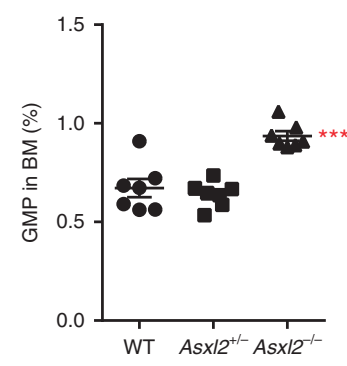

Figure 2 | Altered HSC and myeloid progenitor cell populations in Asx/2 ${ }^{-/-}$mice. (a) Quantification of the per cent $\mathrm{Gr} 1^{+} / \mathrm{Mac1} 1^{+}$cell populations in the PB, BM and spleen of WT, Asx/2+/- and Asx/2-/- mice (6-8 week old, four to five mice per genotype). (b) Flow cytometric analysis of Gr1 ${ }^{+} / \mathrm{Mac}^{+}$ cell populations in PB of representative WT, Asxl2 ${ }^{+/-}$and Asx/2-/- mice. (c) Flow cytometric analysis of $\mathrm{Lin}^{-} \mathrm{cKit}^{+} \mathrm{Sca}^{+}\left(\mathrm{LSK}^{-} \mathrm{and} \mathrm{Lin}^{-} \mathrm{cKit}^{+} \mathrm{Sca}{ }^{-}\right.$ $\left(\mathrm{LKS}^{-}\right.$) compartments in the BM of representative WT, Asx/2 ${ }^{+/-}$and Asx/2-/- mice. (d) Quantification of the per cent LSK population in the total BM cells of each genotype of mice (6-8 week old, seven mice per genotype). (e) Flow cytometric analysis of LT-HSC, short-term (ST)-HSC and MPP compartments in BM LSK cells of representative WT, Asx/2+/- and Asx/2-/- mice. (f) Quantification of the per cent LT-HSC and ST-HSC populations in the total BM cells of each genotype of mice (seven mice per genotype). (g) Flow cytometric analysis of common myeloid progenitor (CMP), GMP and megakaryocyte-erythrocyte progenitor (MEP) cell populations in BM LKS ${ }^{-}$cells of representative WT, Asx/2+/- and Asx/2-/- mice. (h) Quantitative analysis of GMP populations in the total BM cells of each genotype of mice (6-8 week old, seven mice per genotype). Data are presented as mean \pm s.e.m. Comparisons among the groups were performed by one-way analysis of variance. ${ }^{\star} P<0.05,{ }^{\star \star} P<0.01$ and ${ }^{\star \star \star} P<0.001$. 
a

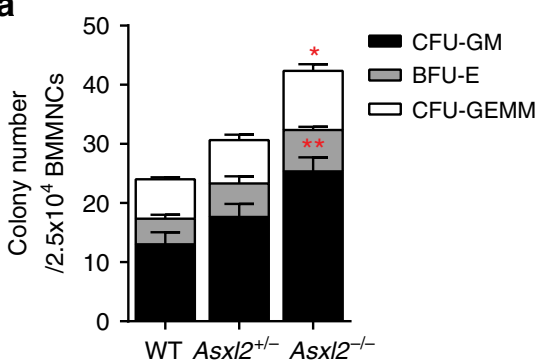

C

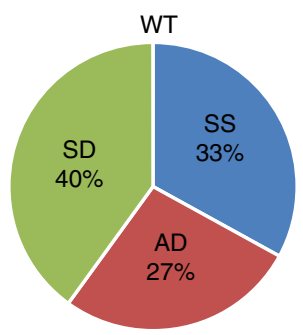

$\mathbf{e}$

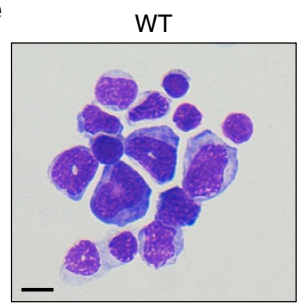

f

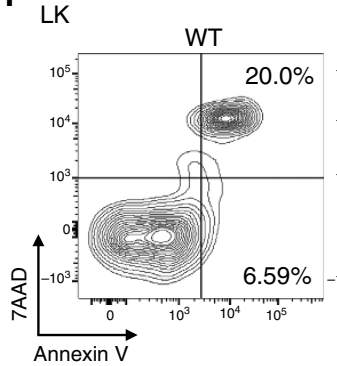

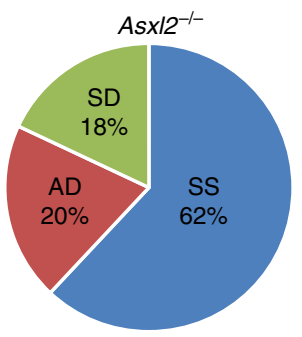

As $\times 12^{-1-}$

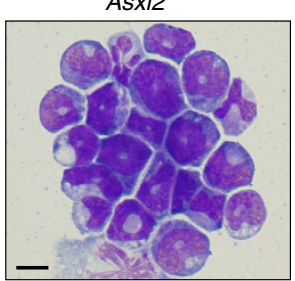

b

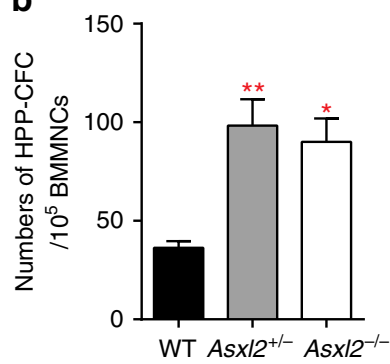

d

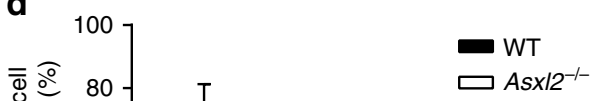

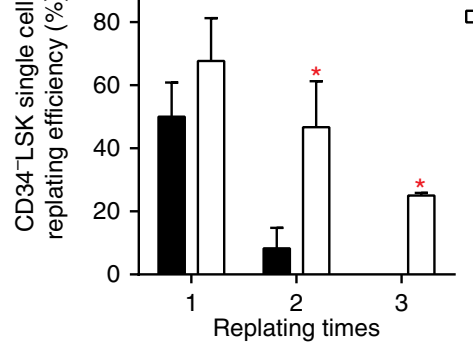

Replating times
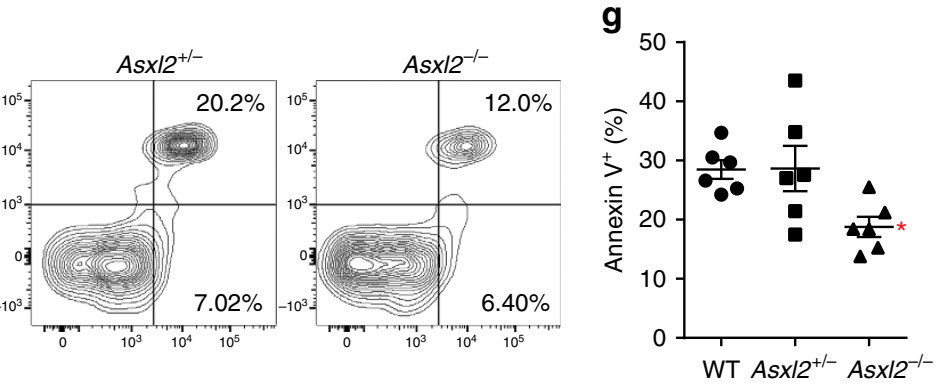

Figure 3 | Loss of Asx/2 alters HSC self-renewal and cell fates. (a) CFUs in BM cells from WT, Asx/2+/- and Asx/2-/- mice (6-8 week old, three mice per genotype) were assessed in semi-solid media in the presence of mouse (m) SCF, mIL-3, human (h) IL-6, mTPO, mouse granulocyte-macrophage colony-stimulating factor (mGM-CSF) and mEPO. BFU-E, burst forming unit-erythrocyte; GEMM, mixed colonies of GM, E, and megakaryocytic cells; GM, granulocytes/macrophages. BMMNCs, bone marrow mononuclear cells. (b) HPP-CFC were determined in the BM of WT, Asx/2+/- and Asx/2-/mice (6-8 week old, three mice per genotype) in the presence of mSCF, human IL6 (hIL-6), hFlt3L, mIL-3, mGM-CSF and mG-CSF. The quantification of large colonies ( $\geq 1 \mathrm{~mm}$ ) was enumerated on the 14th day of cultures. (c) Paired daughter cell assays were performed at single cell level on CD34- LSK cells isolated from WT and Asx/2-/- mice, and each analysed for symmetric self-renewal (SS, blue), asymmetric cell division (AD, red) or symmetric differentiation (SD, green). (d) Single-cell replating assays on the CD34- LSK cells isolated from WT and Asx/2-/- mice were performed to determine replating capacity (6-8 week old, three mice per genotype). (e) Representative images of May-Grünwald-Giemsa-stained cytospin preparations of cells derived from WT and Asx/2 ${ }^{-/}-$CD34- LSK cell cultures. Scale bar, $10 \mu \mathrm{m}$. (f) Apoptosis analysis (Annexin V/7-AAD staining) on freshly isolated $\mathrm{Lin}^{-} \mathrm{cKit}^{+}(\mathrm{LK})$ cells from BM of representative WT, Asx/2+/- and Asx/2-/- mice. (g) Quantification of the apoptotic cells (Annexin $\mathrm{V}^{+}$) within LK cells of BM from WT, Asx/2+/- and Asx/2-/- mice (6-8 week old, six mice per genotype). The colony assay was performed in three replicates. Data are presented as mean \pm s.e.m. Comparisons among more than two groups were performed by one-way analysis of variance test. Comparisons between two experimental groups were performed by two-tailed unpaired Student's $t$-test. ${ }^{\star} P<0.05$ and ${ }^{\star \star} P<0.01$.

Cell-autonomous effect of $A s x l 2$ loss in HSC/HPC functions. To assess whether Asxl2-loss-mediated alteration in HSC/HPC function is cell autonomous, we next performed competitive transplantation assays to examine the repopulating capacity of Asxl2-deficient BM cells (Supplementary Fig. 7a). When the donor cell chimerism was analysed kinetically in the $\mathrm{PB}$ of recipient mice, the CD45.2 cell population remained $\sim 50 \%$ in mice receiving $\mathrm{WT}$ and $A s x l 2^{+/}-\mathrm{BM}$ cells, whereas a transient decline in CD45.2 ${ }^{+}$cells was observed in recipients transplanted with $A s x l 2^{-/-}$BM cells in the first 3 months. Interestingly, the 
a

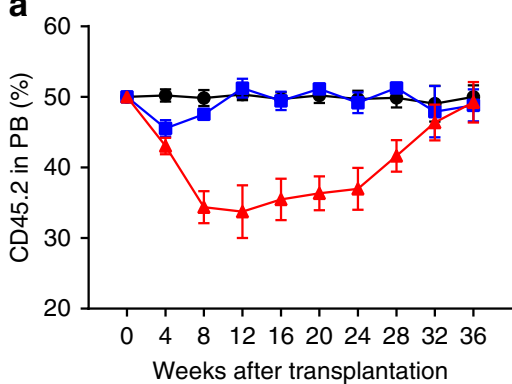

C

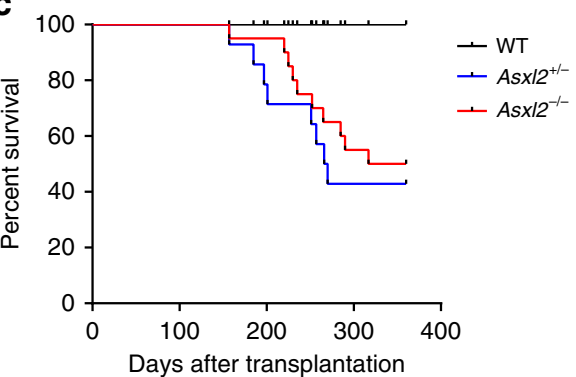

e

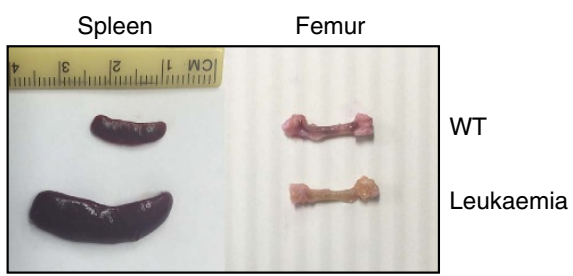

b

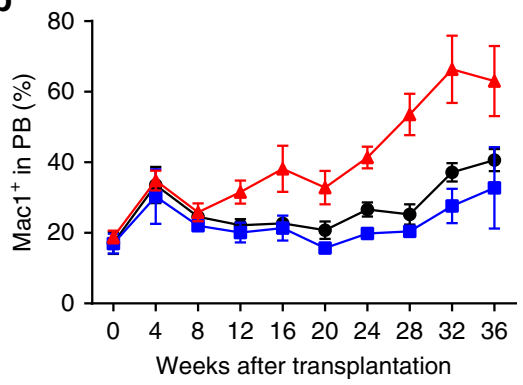

d

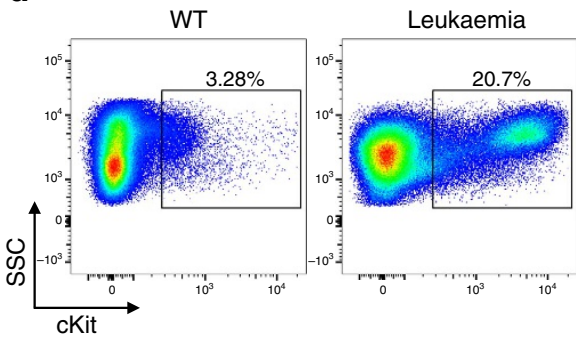

$\mathbf{f}$

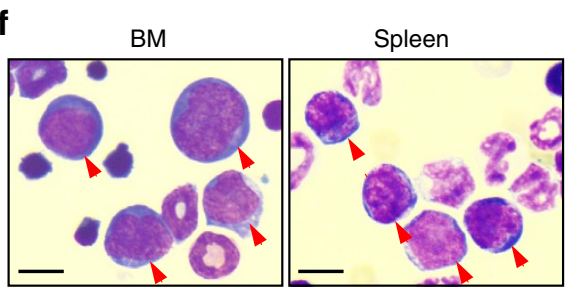

Liver

g
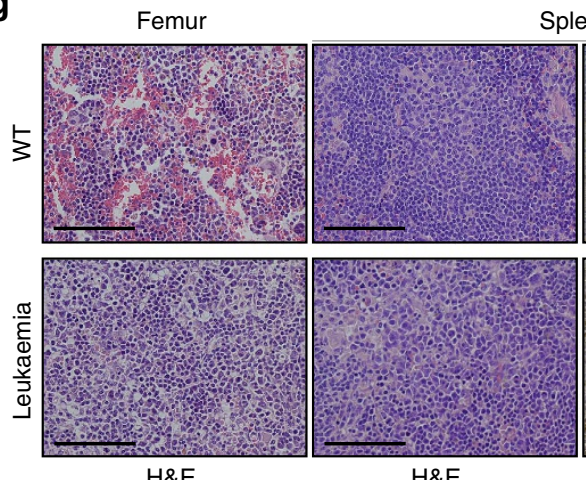

Spleen

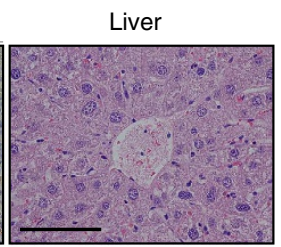

$H \& E$

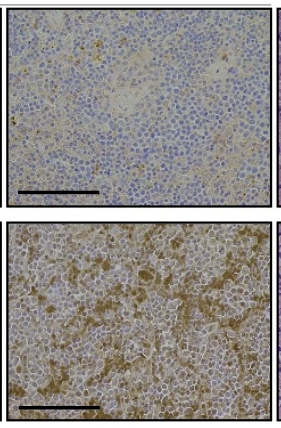

MPO

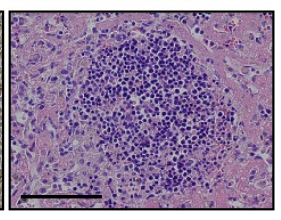

Figure 4 | Cell-autonomous effect of malignant progression in Asx/2 ${ }^{+}$- and Asx/2 ${ }^{-/-}$mice. (a) The kinetics of CD45.2 chimerism in the PB of mice receiving WT, Asxl2 ${ }^{+/-}$or Asxl2 ${ }^{-/-}$BM cells (mean \pm s.e.m., four to eight mice per genotype). (b) The kinetics of percent Mac1 ${ }^{+}$cells in CD45.2 ${ }^{+}$ cells of mice receiving WT, $A s \times 12^{+/-}$or $A s \times 12^{-/-}$BM cells (mean \pm s.e.m., four to eight mice per genotype). (c) Kaplan-Meier curve shows the per cent survival of mice received WT, Asx/2+/- or Asx/2-/- BM cells over time. (d) Flow cytometric analysis of cKit ${ }^{+}$cells in BM. (e) Representative gross appearance of spleens and bones. (f) Representative images of May-Grünwald-Giemsa-stained cytospin preparations of BM and spleen cells from representative leukemic mice receiving Asx/2-/- BM cells. Red arrows indicate myeloblastic cells. Scale bar, $10 \mu \mathrm{m}$. (g) H\&E-stained sections of the femurs, spleens and livers, and MPO-stained spleen sections from a representative leukemic recipient mouse transplanted with $A s \times 12^{-} /-$BM cells and a recipient mouse receiving WT BM cells. Scale bar, $100 \mu \mathrm{m}$.

CD45.2 chimerism was steadily increased in the recipients transplanted with $A s x l 2^{-/-}$BM cells afterwards (Fig. 4a). Furthermore, Asxl2 $2^{-1-} \mathrm{BM}$ cells contributed to a greater proportion of $\mathrm{Macl}^{+}$myeloid cells in the PB than WT BM cells (Fig. 4b).

Five months after transplantation, mice receiving $A s \times l 2^{+/-}$and Asxl2 ${ }^{-/-}$BM cells started to become sick, whereas all the recipients transplanted with WT BM cells were healthy. These Asxl2 $2^{+1-}$ and $A s x l 2^{-1-}$ BM recipient mice exhibited weight loss, pale foot pads and lack of activity, indicative of cachexia symptoms (Supplementary Fig. 7b). These recipients had a greatly shortened lifespan compared with the WT BM recipients (Fig. 4c). Necropsies of the moribund/deceased mice revealed that a fraction of Asxl2 $2^{+/-}$and $A s x l 2^{-I-}$ BM recipients had splenomegaly, severe anemia, decreased platelet counts and significantly higher percentages $(>20 \%)$ of $\mathrm{cKit}^{+}$cells in the BM (Fig. 4d,e and Supplementary Fig. 7c,d). Myeloid blast cells were also seen in the BM, spleen and $\mathrm{PB}$ of these diseased mice (Fig. $4 \mathrm{f}$ and Supplementary Fig. 7e, 
respectively). Histologic analyses showed infiltration of myeloid cells in the BM, spleen and liver (Fig. 4g). Furthermore, flow cytometric analysis revealed increased $\mathrm{Macl}^{+}$population in the $\mathrm{BM}$, spleen and $\mathrm{PB}$, and higher percentages of $\mathrm{cKit}^{+} / \mathrm{Macl}^{+}$cells in the BM and spleen of these diseased mice (Supplementary
Fig. 7f). In contrast, lower percentages of erythroid cells and megakaryocytes were observed in the BM of these diseased recipients (Supplementary Fig. 7g,h). Collectively, these data indicate that a fraction of recipients transplanted with $A s x l 2^{+/-}$ and $A s x l 2^{-/-}$BM cells developed myeloid leukaemia. a

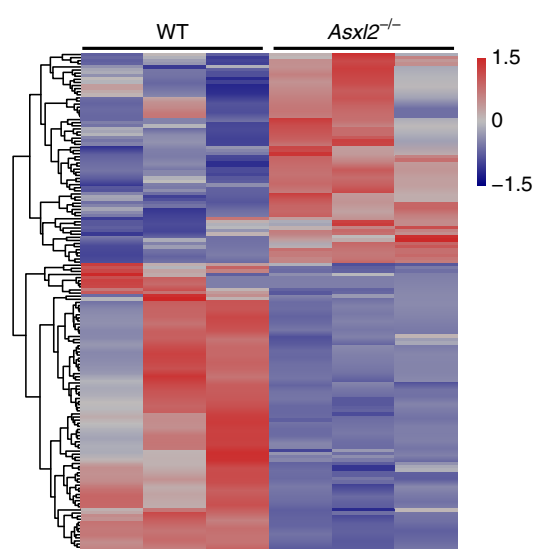

b

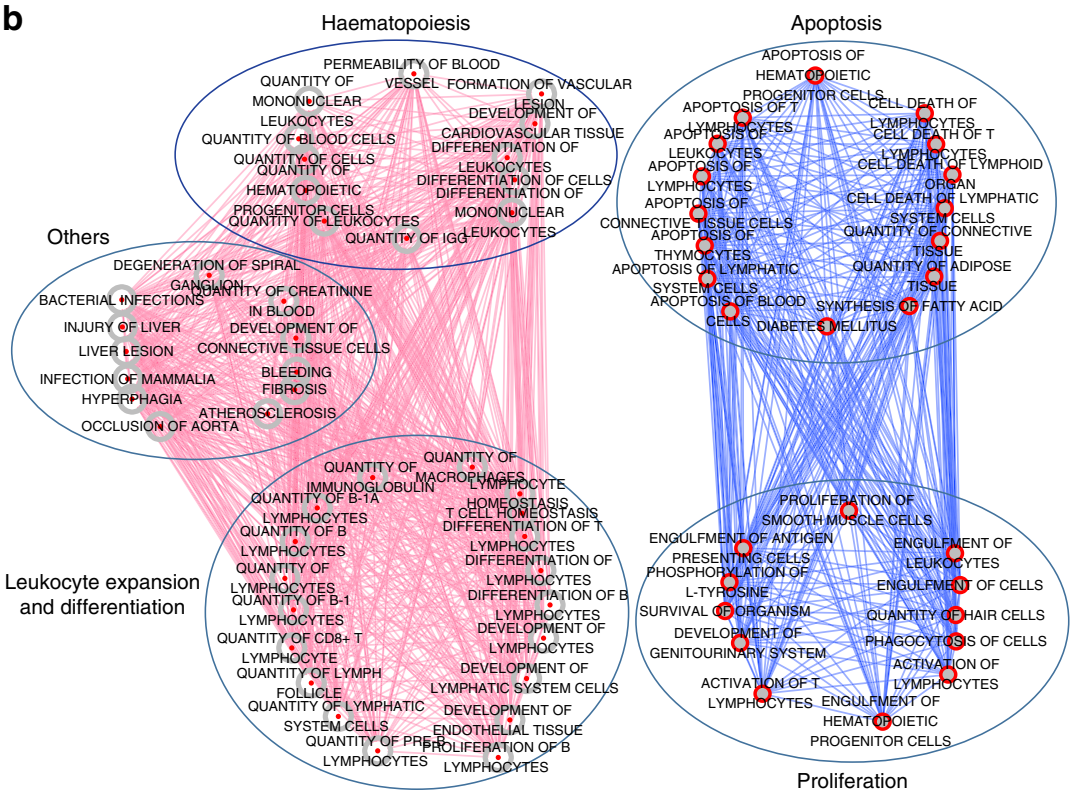

C
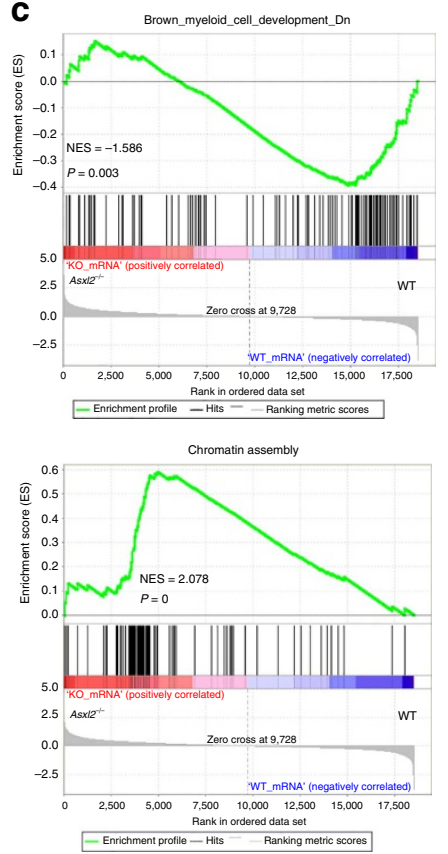
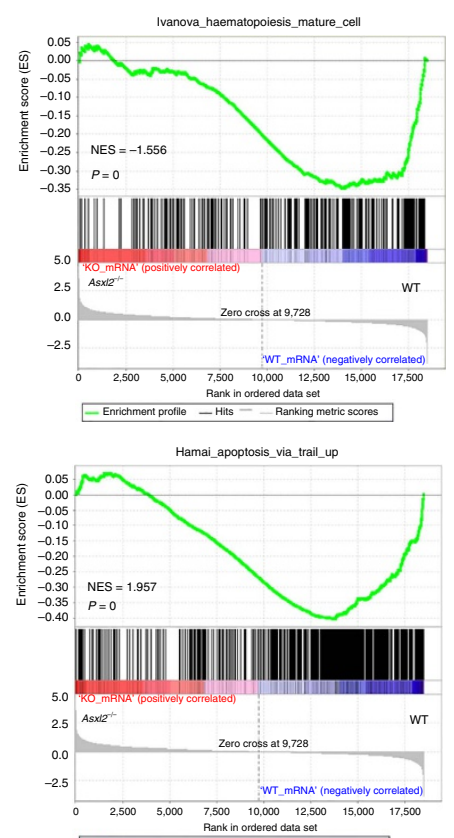

\begin{tabular}{|c|} 
Rankin ordered dalat set \\
\hline Ennichment proflile - Hils - - Ranking metric scores \\
\hline
\end{tabular}
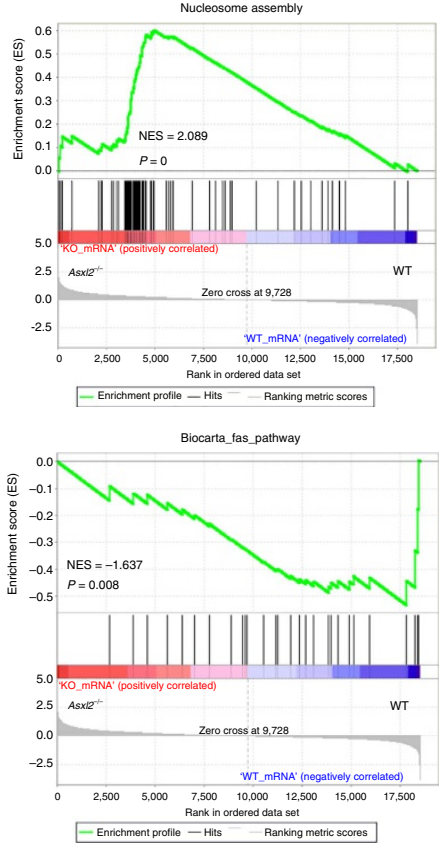
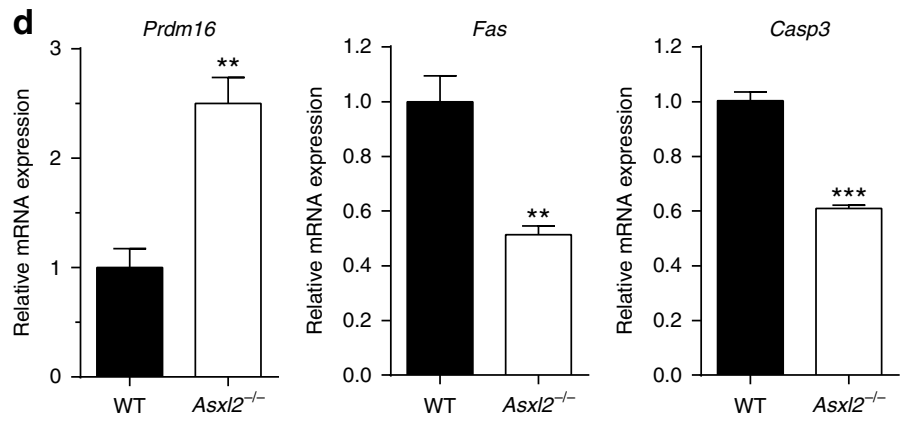
Of note, a fraction of the recipient mice received $A s x l 2^{+/-}$or Asxl2 ${ }^{-1-}$ BM cells developed the MDS-like disease as evidenced by dysplastic features of myeloid lineages, pancytopenia and myeloid infiltration in the spleen and liver (Supplementary Figs $7 \mathrm{e}$ and $8 \mathrm{a}, \mathrm{b}$, and Supplementary Table 3). In addition, a lower proportion of Annexin $\mathrm{V}^{+}$cells in BM LK cells and a higher percentage of Annexin $\mathrm{V}^{+}$cells in $\mathrm{BM} \mathrm{Lin}{ }^{+}$cells were noted in the diseased recipients (Supplementary Fig. 8c).

ASXL2 is required for haematopoietic transcriptional program. PcG proteins are a family of proteins responsible for transcriptional repression during cellular differentiation ${ }^{19}$. To delineate the effect of ASXL2 in the maintenance of HSC self-renewal and cell fates, we performed RNA-seq on LK cells isolated from 6-week-old WT and Asxl2 $2^{-/}$mice. Analysis of RNA-seq data revealed a set of 565 DEGs in Asxl2 ${ }^{-/}$LK cells (Supplementary Dataset $1, P<0.05$, a two-sided test for significance against a null hypothesis), in which 160 DEGs were shown (Fig. 5a, $P<0.05$, false discovery rate $(F D R)<0.25$ and fold change $\geq 1.5$ ). Functional enrichment analysis using Ingenuity Pathway Analysis (IPA) demonstrated that the DEGs were enriched for those related to haematopoiesis, apoptosis and cell differentiation (Fig. 5b and Supplementary Fig. 9a). Consistently, the gene set enrichment analysis (GSEA) showed that the genes with altered expression in Asxl2 $2^{-1-}$ LK cells were enriched for myeloid cell development, haematopoiesis, chromatin/nucleosome assembly, apoptosis and Fas pathway signatures (Fig. 5c).

Asxl2 $2^{-/-}$LK cells had decreased mRNA expression levels of Fas and Casp3, but an increased level of Prdm16 compared with WT cells (Fig. 5d). PRDM16 is required for normal haematopoiesis and highly expressed in a subgroup of AML patients ${ }^{20}$. To assess the effect of $P r d m 16$ overexpression on the haematopoietic phenotypes of $A s x l 2^{-/}$FL cells, Prdm16 was knocked down in Asxl2 ${ }^{-T-}$ FL cells (Supplementary Fig. 9b) and the frequencies of LSK and CFU-C were examined. Prdm16-KD significantly decreased the percentage of LSK cells, as well as the frequency of CFU-C in Asxl2-1- FL cells compared with WT cells (Supplementary Fig. 9c,d).

ASXL2 maintains transcription through histone modifications. It has been shown that deletion of $A s x l 2$ led to reduced global levels of $\mathrm{H} 3 \mathrm{~K} 27 \mathrm{me} 3$ in heart cells $^{8}$, indicating that ASXL2 regulates H3K27 methylation. To determine whether Asxl2 loss would have an impact on the global histone modification states in BM LK cells, we performed western blotting with related histone modification antibodies on WT and $A s x l 2^{-/-}$LK cells. Surprisingly, only a slight reduction of $\mathrm{H} 3 \mathrm{~K} 4 \mathrm{me} 3$ and $\mathrm{H} 3 \mathrm{~K} 27 \mathrm{me} 3$ levels was observed in $A s x l 2^{-1-}$ cells (Supplementary Fig. 10a).

To explore the impact of ASXL2 loss on histone modifications in HSC/HPCs, we performed ChIP-seq using antibodies against H3K4me1, H3K4me2 and H3K27ac. Loss of Asxl2 did not change the enrichment sites of each of the histone marks on the genomic regions (Supplementary Fig. 10b,c). Integrative analysis showed a significantly positive correlation between $\mathrm{H} 3 \mathrm{~K} 27 \mathrm{ac}$ and gene expression in WT and Asxl2 $-/$ LK cells, respectively $(R=0.68$, $P<0.001$; Supplementary Fig. 10d), which is consistent with previous studies ${ }^{21,22}$. Similarly, $R$-values ranging from 0.5 to 0.7 were identified for associations between $\mathrm{H} 3 \mathrm{~K} 4 \mathrm{me} 1 / 2$ and gene expression in WT and $A s x l 2^{-/}-$LK cells, respectively $(P<0.001$, Supplementary Fig. 10d). These data indicate that gene expression is positively associated with $\mathrm{H} 3 \mathrm{~K} 27 \mathrm{ac}$ and $\mathrm{H} 3 \mathrm{~K} 4 \mathrm{me} 1 / 2$.

We further confirmed that the changes in the three histone modifications were positively correlated with the change of mRNA expression $(R=\sim 0.25, P<0.001$, Fig. $6 \mathrm{a}$ and Supplementary Fig. 10e). We identified 1,738, 1,106 and 1,267 regions with differential levels of enrichment between WT and Asxl2 ${ }^{-/-}$LK cells for $\mathrm{H} 3 \mathrm{~K} 27 \mathrm{ac}, \mathrm{H} 3 \mathrm{~K} 4 \mathrm{me} 1$ and $\mathrm{H} 3 \mathrm{~K} 4 \mathrm{me} 2$, respectively. GSEA analysis revealed that genes with increased signals of $\mathrm{H} 3 \mathrm{~K} 27 \mathrm{ac}$, $\mathrm{H} 3 \mathrm{~K} 4 \mathrm{me} 1$ or $\mathrm{H} 3 \mathrm{~K} 4 \mathrm{me} 2$ were concordantly upregulated and genes with decreased signals of $\mathrm{H} 3 \mathrm{~K} 27 \mathrm{ac}, \mathrm{H} 3 \mathrm{~K} 4 \mathrm{mel}$ or $\mathrm{H} 3 \mathrm{~K} 4 \mathrm{me} 2$ were concordantly downregulated on Asxl2 deletion (Supplementary Fig. 11a).

In comparison with $\mathrm{H} 3 \mathrm{~K} 4 \mathrm{me} 1$ and $\mathrm{H} 3 \mathrm{~K} 4 \mathrm{me} 2$, the enrichment of $\mathrm{H} 3 \mathrm{~K} 27 \mathrm{ac}$ had the most significant change at identified regions (Fig. 6b and Supplementary Fig. 11b). Furthermore, the genes with increased and/or decreased H3K27ac signals showed a greater degree of mRNA expression change compared with those with increased or decreased $\mathrm{H} 3 \mathrm{~K} 4 \mathrm{mel} / 2$ signals, respectively (Fig. 6c and Supplementary Fig. 11c; paired $t$-test $P<0.001$ ). Kyoto Encyclopedia of Genes and Genomes (KEGG) pathway analysis showed that genes with differential H3K27ac signals were enriched in signatures of haematopoietic cell lineage, cancer signalling pathway and myeloid leukaemia development (Fig. 6d). IPA analysis further confirmed that genes with altered enrichment levels of $\mathrm{H} 3 \mathrm{~K} 27 \mathrm{ac}$ were enriched in myeloid cell differentiation and apoptosis pathways (Supplementary Fig. 12a-c). Of note, two dysregulated targets (Prdm16 and Fas) were found to be associated with altered histone modifications (Fig. 6e). Meanwhile, two histone deacetylation-related proteins, HDAC1 and HDAC2, were identified to associate with ASXL2 in 293T cells by immunoprecipitation (IP) and western blotting (Supplementary Fig. 13a-c), implying the potential of ASXL2 involvement in the deacetylation of H3K27.

Our data indicate that alteration of H3K27ac enrichment had a greater impact on gene expression compared with $\mathrm{H} 3 \mathrm{~K} 4 \mathrm{me} 1 / 2$ in Asxl2-deficient LK cells. Altogether, these data suggest that ASXL2 loss is associated with changes in gene expression through alterations in $\mathrm{H} 3 \mathrm{~K} 27 \mathrm{ac}$ and $\mathrm{H} 3 \mathrm{~K} 4 \mathrm{mel} / 2$. Further studies to elucidate the mechanism of ASXL2 in regulating HDAC activity are warranted.

\section{Discussion}

Despite the clinical importance of ASXL2 mutations in AML patients with $t(8 ; 21)$, the role of ASXL2 in normal and malignant haematopoiesis remains unknown. Asxl2 is expressed in HSCs and their differentiated HPCs and multiple

\footnotetext{
Figure 5 | Asx/2 deletion alters the expression of genes associated with myeloid cells and apoptosis in LK cells. (a) The heatmap shows the cluster of 160 DEGs between WT and Asx/2-/- LK cells $(P<0.05$, FDR $<0.25$ and fold change $\geq 1.5)$. Red and blue indicate 68 up- and 92 downregulated genes, respectively. (b) Enrichment map is used for visualization of the results of IPA analyses about Diseases and Biological Functions for 565 DEGs ( $<0.05$ ). The network of enriched functional terms (Nodes, $P<0.01$ and $\mid z$-score| $\geq 1.5$ in IPA) is shown. Edges indicate gene overlap between the enriched terms; thickness represents statistical significance of term enrichment. Only edges with a Fisher's exact test nominal $P<10^{-4}$ were visualized. Terms linked by red lines with positive $z$-scores indicate that functional activity is increased, whereas that by blue ones with negative $z$-scores mean decreased activity. Oval indicates functional cluster, which includes relevant terms. (c) GSEA plots showing dysregulated gene expression of myeloid cell development, haematopoiesis, nucleosome/chromatin assembly, apoptosis and Fas pathway signatures in Asxl2 $-/{ }^{-}$LK cells. The normalized enrichment score (NES) and P-value are shown. (d) qPCR showing upregulation of Prdm16 and downregulation of Casp3 and Fas in Asxl2-/ - LK cells compared with WT cells. Data are presented as mean \pm s.e.m. Comparisons between two groups were performed by two-tailed unpaired Student's $t$-test ${ }^{\star \star} P<0.01$ and ${ }^{\star \star \star} P<0.001$.
} 
haematopoietic lineages, suggesting that ASXL2 might play an important role in normal haematopoiesis. In this study, we demonstrated that ASXL2 is required for the normal function of HSCs/HPCs and deletion of Asxl2 in mice increased LT-HSC pool and promoted differentiation towards granulocytic/monocytic lineages.
The majority of the AML patients with somatic ASXL2 mutations are heterozygous and possess a WT allele and an ASXL2 mutant allele, which leads to nonsense/frameshift ${ }^{13}$, indicating that loss-of-function of ASXL2 might be important in the pathogenesis of myeloid malignancies. In the present study, we utilized an Asxl2 knockout mouse model to show that a
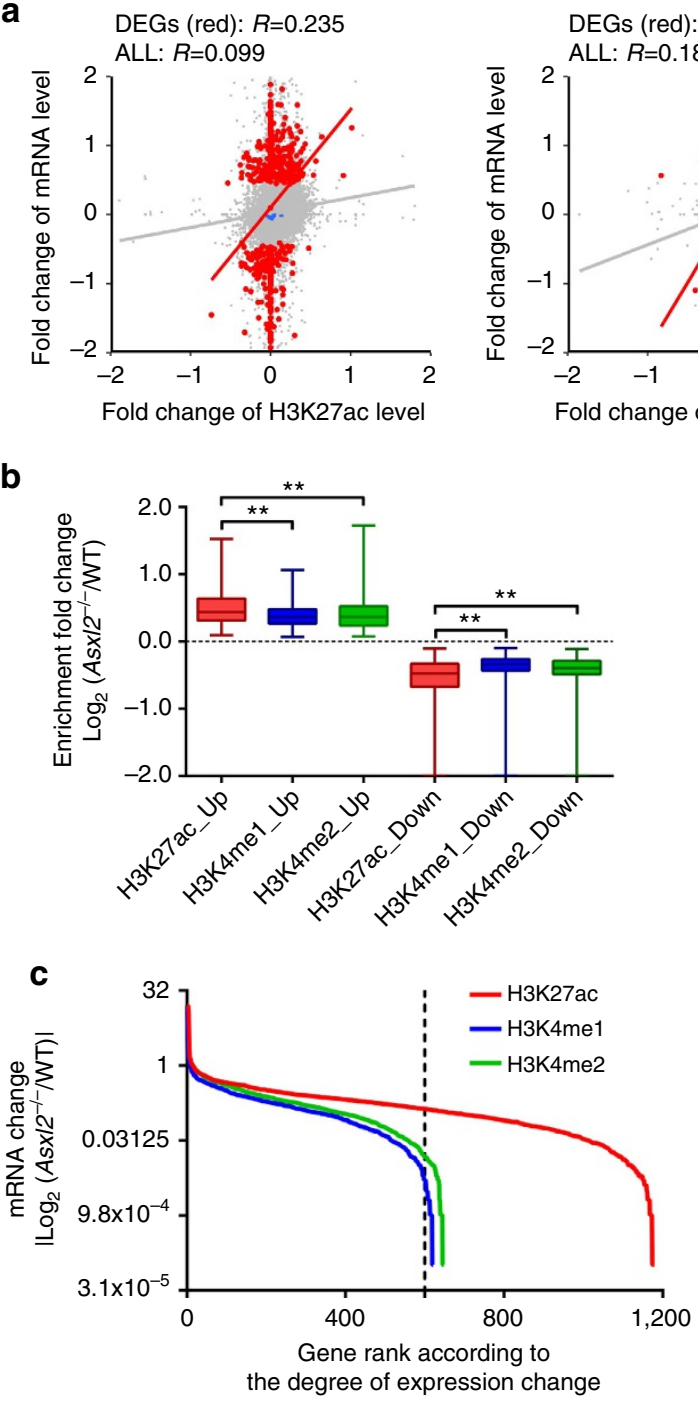

e

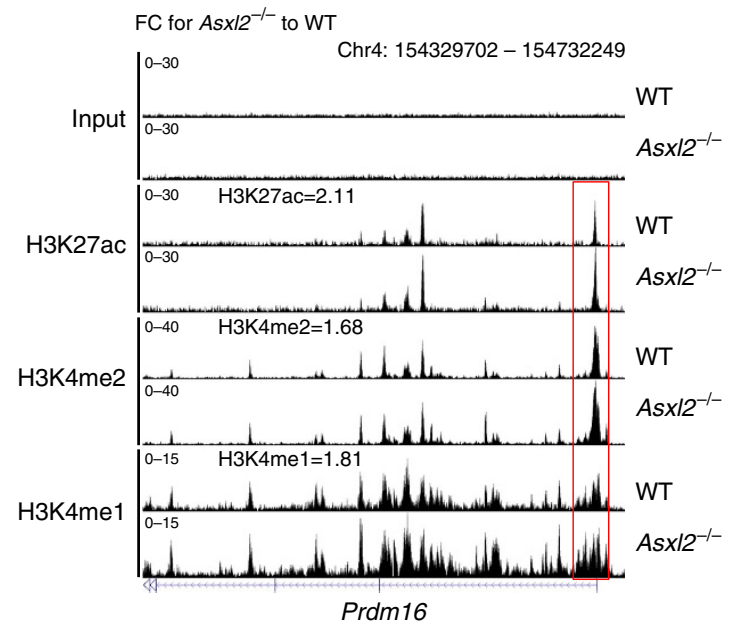

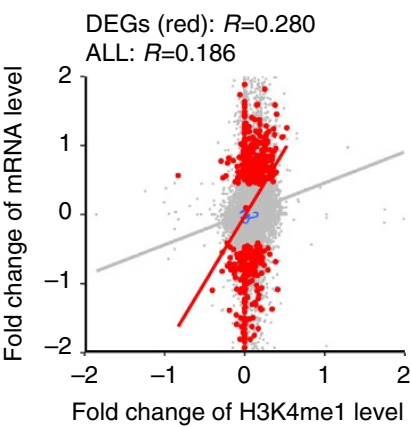
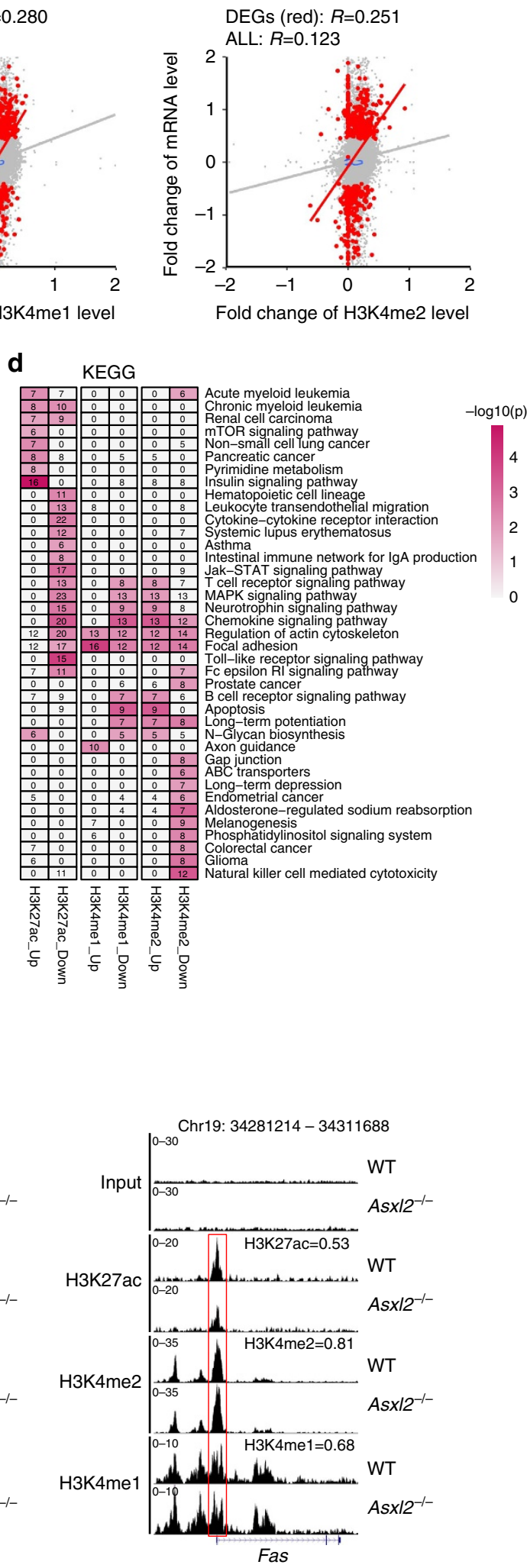
Asxl2 loss in mice leads to dysplastic haematopoiesis, pancytopenia and splenomegaly, resembling an MDS-like disease. Therefore, ASXL2 functions as a tumour suppressor in myelopoiesis.

Normal HSCs are capable of asymmetric division, facilitating both self-renewal and the generation of differentiated progenies. Increased self-renewal potential is one of the hallmarks of cancer $^{23}$. Our paired daughter cell assays revealed that deletion of Asxl2 resulted in an increased symmetric division in HSCs, which probably facilitates the expansion of HSCs in $A s x l 2^{-/-}$mice. Indeed, there was a higher percentage of LT-HSCs in the BM of Asxl2 $2^{-/}$mice compared with WT control mice. Furthermore, deletion of Asxl2 significantly increased the GMP population with skewed differentiation favouring the monocytic/granulocytic lineage. Thus, Asxl2 loss leads to an increased HSC self-renewal and a favourable myeloid differentiation. A recent study by Ye et al. ${ }^{24}$ showed that skewed myeloid differentiation is a prerequisite for leukaemic stem cell formation and AML development. Consistently, skewed expansion of GMP population is associated with higher risks of leukaemic transformation in MDS patients ${ }^{16,25}$. The increased HSC self-renewal and a higher percentage of GMP in Asxl2-deficient mice probably facilitate leukaemogenesis.

Similar to ASXL1 mutations, patients with ASXL2 mutations had a higher incidence of relapse compared with ASXL1/2 WT counterparts $^{13}$, suggesting a critical role of ASXL2 in driving disease progression. In a competitive transplantation assay, As $x 2^{-1}$ HSC/HPCs contributed to a transient decline following a steady ascend in the CD45.2 chimerism in recipients transplanted with $A s x l 2^{-1-}$ BM cells. In addition, Asxl2 $2^{-1-}$ HSC/HPCs gave rise to a greater proportion of myeloid cells in the recipient BM than WT HSC/HPCs. Importantly, both Asxl2 $2^{+-}$ and $A s x l 2^{-/-}$BM recipient mice had a shortened lifespan due to the development of myeloid malignancies. It is possible that the transplantation stress may promote the clonal evolution/ transformation of Asxl2-deficient HSC/HPCs. These competitive transplantation data suggest that Asxl2-loss-mediated haematological phenotype is likely to be cell autonomous. ASXL2 mutations frequently occur in $t(8 ; 21)$ AML and are associated with a higher risk of relapse ${ }^{13}$. Future studies to investigate the synergistic/additive effects of Asxl2 loss plus AML1-ETO in leukaemogenesis are undergoing.

Epigenetic regulation is central to the maintenance of cell identity and disturbance of epigenetic regulation is known to promote tumourigenesis ${ }^{26}$. The GSEA results showed an increased expression of genes related to nucleosome assembly and chromatin assembly signatures in $A s x l 2^{-1-}$ LK cells, revealing a possible mechanism by which ASXL2 regulates haematopoiesis. ASXL family proteins all contain a plant homeodomain domain, as many histone-modifying proteins do, which can bind and recruit epigenetic regulators to modify the histones. As a result, modified histones affect the neighbouring gene transcription. The ASXL2 target genes related to nucleosome and chromatin assembly might be through ASXL2-mediated histone modification regulation.

Histone modifications, such as methylation and acetylation, are a key epigenetic mechanism that regulates gene expression. Our ChIP-seq analysis revealed that loss of Asxl2 altered the levels of histone enhancer marks, including $\mathrm{H} 3 \mathrm{~K} 27 \mathrm{ac}, \mathrm{H} 3 \mathrm{~K} 4 \mathrm{mel}$ and H3K4me2. There was a substantial association between the changes in these histone modifications and gene expression levels. More importantly, we showed that $\mathrm{H} 3 \mathrm{~K} 27 \mathrm{ac}$ genomic enrichment exhibited a higher degree of alteration in Asxl2 $2^{-1-}$ LK cells and had a greater impact on gene expression among the three histone modifications examined. The deregulated genes were enriched for myeloid and haematopoietic cell development, which is consistent with the haematopoietic phenotypes observed in Asxl2-deficient mice. Collectively, these data demonstrate a critical role for ASXL2 in the regulation of haematopoiesis via the maintenance of the histone modification status, in particular H3K27ac. Our IP result showed that ASXL2 indeed interacted with histone deacetylases HDAC1 and HDAC2, suggesting that ASXL2 involves in recruiting/stabilizing HDAC complex to modulate H3K27 acetylation levels. Further investigations to precisely define the mechanism of ASXL2-mediated HDAC activity in haematopoiesis are warranted.

Recently, Lai et al. ${ }^{8}$ showed that deletion of Asxl2 led to reduced global H3K27me3 levels in heart cells. Our study showed that ASXL2 also regulate gene expression via altering the locusspecific levels of $\mathrm{H} 3 \mathrm{~K} 27 \mathrm{ac}$, as well as $\mathrm{H} 3 \mathrm{~K} 4 \mathrm{me} 1 / 2$ in LK cells. As an example, Asxl2 ${ }^{-1-}$ LK cells had significantly upregulated expression of Prmd16. ChIP-seq analysis confirmed the higher H3K27ac signal at the promoter region of $\operatorname{Prdm} 16$, suggesting an association between $\mathrm{H} 3 \mathrm{~K} 27 \mathrm{ac}$ and Prdm16 expression. Interestingly, increased expression of PRDM16 had been reported in a subgroup of AML patients ${ }^{20}$. We and others have reported that ASXL1 is required for polycomb repressive complex 2 binding at target loci in LK cells ${ }^{5-7}$. Our results showed that loss of $A s x l 2$ was associated with alterations in the occupancy of putative enhancers marked by $\mathrm{H} 3 \mathrm{~K} 4 \mathrm{mel} / 2$ and $\mathrm{H} 3 \mathrm{~K} 27 \mathrm{ac}$ in the genome. We, however, cannot exclude a role of other regulatory mechanisms that contribute to the gene alteration, such as insulators.

Collectively, our results demonstrate that ASXL2 plays an important role in normal haematopoiesis and Asxl2 loss in mice leads to myeloid malignancies. The Asxl2 knockout mice present an ideal model for unveiling the mechanisms underlying the Asxl2-loss-mediated multiple-step pathogenesis of myeloid malignancies and for testing novel therapeutic agents for myeloid malignant patients with ASXL2 mutations. ASXL1 and ASXL2 probably have both overlapping and unique functions in normal and malignant haematopoiesis. Future work is warranted to

\footnotetext{
Figure 6 | ASXL2 maintains transcriptional programs through H3K27ac and H3K4me1/2. (a) Scatter plots with kernel density estimation showing correlations between change in $\mathrm{H} 3 \mathrm{~K} 27 \mathrm{ac}$ and $\mathrm{H} 3 \mathrm{~K} 4 \mathrm{me} 1 / 2$, and changes in gene expression in WT and Asx/2-/ - LK cells. Grey and red points represent all expressed genes and DEGs, respectively. Solid line, linear fit. Spearman's correlation coefficient $R$-values are shown, $P<10^{-7}$ for all correlation tests. $X$ and $Y$ axes show the $\log _{2}$ transformed fold change $\left(\log _{2} \mathrm{FC}\right)$ for each histone enrichment and mRNA expression level, respectively. (b) Boxplot displaying enrichment difference in H3K27ac, H3K4me1 and H3K4me2 at increased (up) and decreased (down) enrichment regions between WT and Asx/2- / - LK cells. $Y$ axis shows the $\log _{2} \mathrm{FC}$ in histone modification. ${ }^{\star \star} P<0.001$ (unpaired Student $t$-test). (c) Line plots representing mRNA expression differences for genes with increased and/or decreased signals in H3K27ac, H3K4me1 and H3K4me2 between WT and Asx/2-/ ${ }^{-}$LK cells. $X$ axis and $Y$ axis with log 2 scale show the rank of genes with differential signals according to the degree of expression change in decreasing order. (d) Heatmap representing KEGG pathway enriched by the genes with differentially enriched regions (DERs) of H3K27ac, H3K4me1 and H3K4me2. Row and column represent KEGG pathway and gene set, respectively. Colour scale indicates enrichment significance. The number in each cell shows the number of enriched genes in this pathway. Up and down represent genes with increased and decreased signals, respectively. (e) Representative genome browser tracks showing H3K27ac, H3K4me1 and H3K4me2 enrichment on promoter regions of Prdm16 and Fas, as well as their fold change between WT and Asx/2-/- LK cells. The red box indicates DER.
} 
decipher this critical question and unveil the roles of ASXL1 and ASXL2 mutations in the pathogenesis of myeloid malignancies.

\begin{abstract}
Methods
Asx/2-null-deficient mouse model and reagents. The targeting strategy of the Asxl2 $2^{-1-}$ mice was reported previously by Baskind et al. ${ }^{15}$. Briefly, the gene trap cassette was integrated into the first intron of Asxl2; the exact site of integration was 5,016 bp downstream of exon 1 . All studies were conducted in accordance with the regulatory guidelines by the Institutional Animal Care and Use Committee at University of Miami Miller School of Medicine. Chemicals were obtained from Sigma (St Louis, MO) unless otherwise indicated.
\end{abstract}

Phenotypic analyses of the haematologic system in mice. PB was collected by retro-orbital bleeding and was subjected to an automated blood count (Hemavet System 950FS). For morphological and lineage differential analysis, PB smears were subjected to May-Grünwald-Giemsa staining. Morphological analysis of BM and spleen cells were performed on cytospins followed by May-Grünwald-Giemsa staining. For histopathology analyses, femurs were fixed in formaldehyde and demineralized in a solution of 10\% EDTA for 1-2 weeks. The specimens and other soft tissues (spleens and livers) were dehydrated using ethanol and cleared in xylenes. The specimens were then embedded in melted paraffin and allowed to harden. Thin sections $(4-5 \mu \mathrm{m})$ were cut and floated onto microscope slides. For routine assessment, slides were stained with haematoxylin and eosin. For MPO staining, the tissue was rehydrated followed by heat-induced epitope retrieval, peroxidase and serum blocking. Samples were then incubated with MPO antibody (R\&D, MAB3174) overnight at $4{ }^{\circ} \mathrm{C}$ followed by staining with the biotinylated second antibody. Slides were visualized under a Nikon TE2000-S microscope. Images were taken by a QImaging camera and QCapture-Pro software (Fryer Company Inc.).

Flow cytometric analysis, cell sorting and HSC/HPC assay. Total white blood cells were obtained after lysis of $\mathrm{PB}$ with red cell lysis buffer. Single-cell suspensions from the BM, spleen, liver and PB were stained with panels of fluorochrome-conjugated antibodies (Supplementary Table 1). Flow cytometric analysis of HSC/HPCs was performed as previously described ${ }^{6}$. Dead cells were excluded by 4,6-diamidino-2phenylindole staining. The analyses were performed using a BD FACS Canto II or LSR Fortessa flow cytometer. For cell apoptosis analysis, freshly isolated BM cells were stained with lineage/cKit antibodies and PE-Annexin V/7-AAD apoptotic kit (BD Biosciences) according to the protocol and analysed with the $\mathrm{Lin}^{+}$subpopulation and $\mathrm{Lin}^{-} \mathrm{cKit}^{+}$(LK) subpopulation. For cell cycle analysis, BM cells were labelled with BrdU for $45 \mathrm{~min}$ in vitro, stained with surface markers, treated with DNase and finally stained with BrdU and 7-AAD. All data were analysed by FlowJo-V10 software. For LK cell selection, magnetic-activated cell sorting was applied. BM cells were first sorted with lineage depletion beads (Miltenyi Biotec, Bergisch Gladbach, Germany) and the lineage-negative cells were then sorted with cKit (CD117) beads. The purity of selected LK cells was routinely over $95 \%$. For CFU assays, BM or spleen cells were plated in triplicate in methylcellulose medium (Methocult M3134, StemCell Technologies) supplemented with mouse $(\mathrm{m})$ stem cell factor (mSCF, $100 \mathrm{ng} \mathrm{ml}^{-1}$ ), human interleukin 6 (IL-6, $50 \mathrm{ng} \mathrm{ml}^{-1}$ ), IL 3 (mIL-3, $5 \mathrm{ng} \mathrm{ml}^{-1}$ ), erythropoietin (EPO, $4 \mathrm{U} \mathrm{ml}^{-1}$ ), thrombopoietin (mTPO, $100 \mathrm{ng} \mathrm{ml}^{-1}$ ) and mouse granulocytemacrophage colony-stimulating factor $\left(10 \mathrm{ng} \mathrm{ml}^{-1}\right.$, Peprotech), and scored on day 7 of the cultures ${ }^{27}$.

Paired daughter cell assay. To examine the frequency of HSCs self-renewal and differentiation capability, we performed paired daughter cell assays ${ }^{28}$. Single CD34 $4^{-}$LSK cells from BM of WT and Asxl2 ${ }^{-1-}$ mice were clone sorted into 96-well plates. The cells were maintained in RPMI1640 media supplemented with $\mathrm{mSCF}\left(100 \mathrm{ng} \mathrm{ml}^{-1}\right)$ and $\mathrm{mTPO}\left(50 \mathrm{ng} \mathrm{ml}^{-1}\right)$. After the first cell division, the two daughter cells were separated, one per well for an additional 12 days in the media supplemented with $\mathrm{mSCF}, \mathrm{mTPO}, \mathrm{mEPO}$, mIL-3 and granulocyte colonystimulating factor (mG-CSF). The self-renewal and differentiation capabilities of cultured CD $34^{-}$LSK cells were determined by morphological analyses microscopically following haematoxylin and eosin staining. A total of 288 single cells (three 96-well plates) was analysed to calculate the percentage of symmetric/ asymmetric cell divisions.

Single-cell colony assay. To explore the proliferative potential of single LT-HSC, we performed the single-cell colony assay as previously described ${ }^{29}$. Single CD34- LSK cells were sorted into 96-well plates with $10 \%$ fetal bovine serum, $1 \% \mathrm{BSA}, 2 \mathrm{mM} \mathrm{L}$-glutamine and $5 \times 10^{5} \mathrm{M} 2$ - $\beta$-mercaptoethanol in $\alpha$-MEM, supplemented with cytokines cocktails, including $\mathrm{mSCF}\left(20 \mathrm{n} \mathrm{ml}^{-1}\right), \mathrm{mTPO}$ $\left(20 \mathrm{ng} \mathrm{ml}^{-1}\right), \mathrm{mIL}-3\left(10 \mathrm{ng} \mathrm{ml}^{-1}\right)$ and $\mathrm{mEPO}\left(4 \mathrm{U} \mathrm{ml}^{-1}\right)$. The colonies were scored after 7 days and the progenitors of individual colonies were collected and replated in a new 96-well plate. Cytospin slides were prepared from individual colonies followed by May-Grünwald-Giemsa staining. Antibodies used for the single cell sorting were shown in Supplementary Table 1.

HPP-CFC assay. Bone marrow mononuclear cells $\left(1 \times 10^{5}\right)$ from WT, Asxl2 $2^{+/-}$ or Asxl2 ${ }^{-1-}$ mice were cultured in triplicate in $35-\mathrm{mm}$ plates using soft agar for the growth of HPP CFCs in the presence of mSCF $\left(100 \mathrm{ng} \mathrm{ml}^{-1}\right)$, human IL-6 $\left(50 \mathrm{ng} \mathrm{ml}^{-1}\right)$, hFlt3L $\left(10 \mathrm{ng} \mathrm{ml}^{-1}\right)$, mIL-3 $\left(10 \mathrm{ng} \mathrm{ml}^{-1}\right)$, mouse granulocytemacrophage colony-stimulating factor $\left(10 \mathrm{ng} \mathrm{ml}^{-1}\right)$ and $\mathrm{mG}-\mathrm{CSF}\left(10 \mathrm{ng} \mathrm{ml}^{-1}\right)$. Cultures were incubated at $5 \% \mathrm{CO}_{2}, 5 \% \mathrm{O}_{2}$ and scored by indirect microscopy on day 14 for HPP-CFCs.

Competitive repopulation assay. The competitive repopulation assay was performed by transplanting BM cells $\left(1 \times 10^{6}, \mathrm{CD} 45.2\right)$ from WT, Asxl2 ${ }^{+/-}$or Asxl2 ${ }^{-1-}$ mice plus the competitor BM cells $\left(1 \times 10^{6}\right.$, CD45.1) from B6.SJL mice, into lethally irradiated $(9.5 \mathrm{~Gy})$ recipients $(\mathrm{CD} 45.1)$ by tail vein injection. Mice were monitored daily for signs of disease development.

Lentiviral transduction. The RNAi Consortium (TRC)-based Prdm16 short hairpin RNA lentiviral vector (GE Healthcare Dharmacon, Inc.) were transfected into HEK 293T cells (ATCC) to package viruses. The supernatant was collected $48 \mathrm{~h}$ after transfection. The FL cells from WT and Asxl2 ${ }^{-1-}$ embryos (E12.5) were transduced with the packaged viruses and selected with puromycin. FL cells were collected into different dishes for colony-forming assays and liquid cultures.

qPCR and RNA-seq analysis. Total RNA was isolated from BM LK cells of each mouse genotype and treated with RNase-free DNase to remove contaminating genomic DNA. First-strand complementary DNA was synthesized. qPCR was performed using Fast SYBR Green master mix (Applied Biosystems). PCR amplifications were performed in triplicate for each gene of interest along with parallel measurements of Actin cDNA (internal control). To confirm specific amplification of the desired PCR product, melting curves were analysed and PCR products were separated on a $2 \%$ agarose gel. The primers used for the amplification of each gene are shown in Supplementary Table 2.

Total RNA was isolated from mouse Asxl2 ${ }^{-1-}$ and WT LK cells following standard protocol with TRIZol reagent (Life Technologies) followed by RNA library preparation with the Illumina TruSeq strand-specific mRNA sample preparation system. All RNA-seq libraries were sequenced with a read length of single-end $75 \mathrm{bp}$ using the Illumina NextSeq 500 and final of over 45 million reads per sample.

RNA-seq reads were aligned to the mouse genome reference sequence (GRCm38/mm10) and RefSeq annotation (Release 23 May 2014) obtained from UCSC using TopHat $(\mathrm{v} 2.0 .9)^{30}$ with a parameter of --max-multihits = 1. FastQC (v0.10.1) (http://www.bioinformatics.babraham.ac.uk/projects/fastq) and RseQC (v2.3.8) software $^{31}$ were used to check the quality of the raw reads. The quality score of each read reached an average of 25 . The rates of overall mapped reads reached approximately $95 \%$. Cufflink software (v2.0.2) $)^{30}$ was used to quantify the gene expression values by calculating the FPKM value (the fragment per kilobase of transcript per million fragments mapped). Cuffdiff ${ }^{30}$ was used to detect the DEGs and DEGs with a cutoff of $P<0.05$ were selected for further pathway analyses. Expression profiles for all genes including DEGs are shown in Supplementary Dataset 1. GSEA analysis ${ }^{32}$ was performed with gene signatures in GESA/MSigDB v5.1, including KEGG pathway signatures and Gene Ontology signatures. Enriched gene sets or pathways were selected using a cutoff of FDR $<0.25$.

Western blotting and IP assays. IP was performed ${ }^{33}$ using nuclear fraction buffer and antibodies (ASXL2 at a dilution of 1:500, Santa Cruz, sc-169972; HDAC1 at a dilution of 1:500, ThermoFisher Scientific, PA1-860; and HDAC2 at a dilution of 1:2,000, PA1-861). After washing with IP buffer (20 mM Tris-HCl, pH 7.5, $150 \mathrm{mM}$ $\mathrm{NaCl}, 1 \%$ Triton X-100, $5 \mathrm{mM}$ EDTA, $2 \mathrm{mM}$ sodium orthovanadate, $1 \mathrm{mM}$ phenylmethylsulfonyl fluoride, $2 \mathrm{mM} \mathrm{NaF}$ and protease inhibitor cocktail (Roche)) for four times, the associated proteins were collected for western blot analysis ${ }^{34}$. The histone modifications and protein levels were determined using antibodies at dilutions of 1:800 against H3K4me3 (Abcam, ab8580) and H3K27me3 (ab6002).

Chromatin immunoprecipitation. LK cells of each mouse genotype were fixed with $1 \%$ formaldehyde for $15 \mathrm{~min}$ and quenched with $0.125 \mathrm{M}$ glycine. Chromatin was isolated by the addition of lysis buffer, followed by shearing with Bioruptor Pico with water cooler (Diagenode, Seraing, Belgium). The DNA was sheared to an average length of 300-500 bp. Genomic DNA regions of interest were isolated using antibodies against H3K27ac (Diagenode, C15410196), H3K4me1 (C15410194) and H3K4me2 (Abcam, ab32356). Complexes were washed, eluted from the beads with SDS buffer and subjected to RNase and proteinase $\mathrm{K}$ treatment. Crosslinks were reversed by incubation overnight at $65^{\circ} \mathrm{C}$ and $\mathrm{ChIP}$ DNA was purified by phenol-chloroform extraction and ethanol precipitation. After the measurement of DNA concentration with Qubit3.0, the libraries were prepared and sequenced on an Illumina NextSeq 500.

ChIP sequencing and data analysis. ChIP-seq libraries for H3K27ac, H3K4me1 and $\mathrm{H} 3 \mathrm{~K} 4 \mathrm{me} 2$ were prepared and sequenced with a read length of single-end $75 \mathrm{bp}$ on NextSeq 500 and a final of over 45 million reads per sample. The sequence reads were aligned to the mouse genome (GRCm38/ $\mathrm{mm} 10$ ) using the bowtie2 (v2.1.0) algorithm with default settings. The rates of overall mapped reads were at least $95 \%$. Reads that aligned to the same position and strand were only counted once to 
avoid potential PCR bias. Finally, 40-70\% of total reads were kept for further analysis. Enrichment peaks of histone modifications were called using the MACS $(\mathrm{v} 14)^{35}$ with parameters of --fe-min $=1$--fe-max $=40$ and filtered according to FDR $\leq 0.01$, mean signal $\geq 0.02$ and enrichment fold $\geq 2.5$. The resulting genomic signals in wiggle files were visualized using UCSC genome browser (http://genome.ucsc.edu/). MAnorm ${ }^{36}$ was used to identify differential enrichment regions for each histone modification between $A s x l 2^{-1-}$ and WT LK cells, with $P \leq 0.01$ and read count after normalization to reads per million (RPM) $\geq 32$. Peaks, binding sites and differential enrichment regions were assigned to its nearest genes within $100 \mathrm{~kb}$ using RefSeq annotation (Release 23 May 2014) from UCSC. Peaks overlapping with the regions from upstream $2 \mathrm{~kb}$ to downstream $2 \mathrm{~kb}$ of TSS (TSS $\pm 2 \mathrm{~kb}$ ) were allocated to the promoter. Peaks overlapping with gene body rather than promoter were allocated to gene body. The remaining intergenic peaks were assigned to both the nearest $5^{\prime}$ - and $3^{\prime}$-genes within its $100 \mathrm{~kb}$. If no gene was found within $100 \mathrm{~kb}$ of an intergenic peak, it was not assigned to any gene. The binding sites and differential enriched regions in H3K27ac and H3K4me1/2 are shown in Supplementary Data set 2, respectively. Peak overlapping and merging were done with multovl (v1.2) ${ }^{37}$. ChIP-seq read counts were normalized to reads per kilobase per million reads and combined into one matrix with deeptools ${ }^{38}$ (http://deeptools.ie-freiburg.mpg.de/) for heatmap visualization. Given $80 \%$ of histone modification peaks were located in regions ranging from upstream $10 \mathrm{~kb}$ to downstream $10 \mathrm{~kb}$ of gene body (gene $\pm 10 \mathrm{~Kb}$ ), the ChIP-seq reads within Gene $\pm 10 \mathrm{~kb}$ were counted using cisgenome (v2) ${ }^{39}$ with a parameter of $-\mathrm{e} 200$ and normalized to RPM for integrational analysis of ChIP-seq and gene expression data $^{39}$. For each gene, enrichment fold was defined as $\log _{2}$ transformed RPM in ChIP divided by RPM in input, $\log _{2}$-(ChIP/input) and revised to zero when it was smaller than zero. This enrichment fold matrix was used to do GESA analysis of DEGs (http://software.broadinstitute.org/gsea) ${ }^{32}$, to examine whether DEGs have differential enrichment signals of H3K27ac and H3K4me1/2. All figures were visualized in $R$ using ggplot2 and pheatmap and GraphPad (v5.0).

Pathway and biological functional enrichment analyses. The identified DEGs and genes with the differential signals of H3K27ac, H3K4mel or H3K4me2 in Asxl2 ${ }^{-1-}$ LK cells compared with WT cells were used for the pathway and biological function enrichment analyses with the DAVID Bioinformatics Resources v6.7 (https://david.ncifcrf.gov/ $/)^{40}$ and IPA. Enriched KEGG pathways $(P<0.05$ and FDR $\leq 0.25)$ using DAVID were shown in heatmap. Enriched terms with the association to diseases and biological functions $(P<0.05$ and $\mid z$-score $\mid \geq 1.5)$ using IPA were shown in Enrichment Map for function clusters ${ }^{41}$

Statistical analysis. Differences between experimental groups were determined by the Student's $t$-test or analysis of variance followed by Newman-Keuls multiple comparison tests as appropriate. $P<0.05$ is considered significant. For the in vivo experiment, sample size chosen was based on the generalized linear model with Bonferroni multiple comparison adjustments, with the proposed sample size of at least five mice per group/genotype. Animals were randomly assigned to each study. For all in vitro experiments, at least three independent experiments with more than three biological replicates for each condition/genotype were performed to ensure adequate power.

Data availability. Raw data generated from RNA-seq and ChIP-seq have been deposited in the Genome Sequence Archive (http://gsa.big.ac.cn/) in BIG Data Center ${ }^{42}$ under accession number PRJCA000259. All other remaining data are available within the Article and Supplementary Files, or available from the authors upon request.

\section{References}

1. Li, S., Mason, C. E. \& Melnick, A. Genetic and epigenetic heterogeneity in acute myeloid leukemia. Curr. Opin. Genet. Dev. 36, 100-106 (2016).

2. Sauvageau, M. \& Sauvageau, G. Polycomb group genes: keeping stem cell activity in balance. PLoS Biol. 6, el13 (2008).

3. Fisher, C. L., Randazzo, F., Humphries, R. K. \& Brock, H. W. Characterization of Asxl1, a murine homolog of additional sex combs, and analysis of the Asxlike gene family. Gene 369, 109-118 (2006).

4. Cao, R. et al. Role of histone H3 lysine 27 methylation in Polycomb-group silencing. Science 298, 1039-1043 (2002).

5. Abdel-Wahab, O. et al. ASXL1 mutations promote myeloid transformation through loss of PRC2-mediated gene repression. Cancer Cell 22, 180-193 (2012).

6. Wang, J. et al. Loss of Asxll leads to myelodysplastic syndrome-like disease in mice. Blood 123, 541-553 (2014).

7. Abdel-Wahab, O. et al. Deletion of Asxl1 results in myelodysplasia and severe developmental defects in vivo. J. Exp. Med. 210, 2641-2659 (2013).

8. Lai, H. L. et al. Maintenance of adult cardiac function requires the chromatin factor Asxl2. J. Mol. Cell Cardiol. 53, 734-741 (2012).

9. Gelsi-Boyer, V. et al. Mutations of polycomb-associated gene ASXL1 in myelodysplastic syndromes and chronic myelomonocytic leukaemia. Br. J. Haematol. 145, 788-800 (2009).
10. Boultwood, J. et al. Frequent mutation of the polycomb-associated gene ASXL1 in the myelodysplastic syndromes and in acute myeloid leukemia. Leukemia $\mathbf{2 4}$, 1062-1065 (2010).

11. Abdel-Wahab, O. et al. Genetic analysis of transforming events that convert chronic myeloproliferative neoplasms to leukemias. Cancer Res. 70, 447-452 (2010).

12. Huether, R. et al. The landscape of somatic mutations in epigenetic regulators across 1,000 paediatric cancer genomes. Nat. Commun. 5, 3630 (2014).

13. Micol, J. B. et al. Frequent ASXL2 mutations in acute myeloid leukemia patients with $\mathrm{t}(8 ; 21)$ /RUNX1-RUNX1T1 chromosomal translocations. Blood 124, 1445-1449 (2014).

14. Faber, Z. J. et al. The genomic landscape of core-binding factor acute myeloid leukemias. Nat. Genet. 48, 1551-1556 (2016).

15. Baskind, H. A. et al. Functional conservation of Asxl2, a murine homolog for the Drosophila enhancer of trithorax and polycomb group gene Asx. PLoS ONE 4, e4750 (2009).

16. Will, B. et al. Stem and progenitor cells in myelodysplastic syndromes show aberrant stage-specific expansion and harbor genetic and epigenetic alterations. Blood 120, 2076-2086 (2012).

17. Yoder, M. C., Du, X. X. \& Williams, D. A. High proliferative potential colonyforming cell heterogeneity identified using counterflow centrifugal elutriation. Blood 82, 385-391 (1993).

18. Cicalese, A. et al. The tumor suppressor p53 regulates polarity of self-renewing divisions in mammary stem cells. Cell 138, 1083-1095 (2009).

19. Simon, J. A. \& Tamkun, J. W. Programming off and on states in chromatin: mechanisms of Polycomb and trithorax group complexes. Curr. Opin. Genet. Dev. 12, 210-218 (2002).

20. Matsuo, H., Goyama, S., Kamikubo, Y. \& Adachi, S. The subtype-specific features of EVI1 and PRDM16 in acute myeloid leukemia. Haematologica 100, el16-e117 (2015).

21. Li, R. et al. Obesity, rather than diet, drives epigenomic alterations in colonic epithelium resembling cancer progression. Cell Metab. 19, 702-711 (2014).

22. Dong, X. \& Weng, Z. The correlation between histone modifications and gene expression. Epigenomics 5, 113-116 (2013).

23. Hanahan, D. \& Weinberg, R. A. The hallmarks of cancer. Cell 100, 57-70 (2000).

24. Ye, M. et al. Hematopoietic differentiation is required for initiation of acute myeloid leukemia. Cell Stem Cell 17, 611-623 (2015).

25. Goardon, N. et al. Coexistence of LMPP-like and GMP-like leukemia stem cells in acute myeloid leukemia. Cancer Cell 19, 138-152 (2011).

26. Tarayrah, L. \& Chen, X. Epigenetic regulation in adult stem cells and cancers. Cell Biosci. 3, 41 (2013).

27. Shi, H. et al. ASXL1 plays an important role in erythropoiesis. Sci. Rep. 6, 28789 (2016).

28. Takano, H., Ema, H., Sudo, K. \& Nakauchi, H. Asymmetric division and lineage commitment at the level of hematopoietic stem cells: inference from differentiation in daughter cell and granddaughter cell pairs. J. Exp. Med. 199, 295-302 (2004).

29. Ema, H. et al. Adult mouse hematopoietic stem cells: purification and single-cell assays. Nat. Protoc. 1, 2979-2987 (2006).

30. Trapnell, C. et al. Differential gene and transcript expression analysis of RNAseq experiments with TopHat and Cufflinks. Nat. Protoc. 7, 562-578 (2012).

31. Wang, L., Wang, S. \& Li, W. RSeQC: quality control of RNA-seq experiments. Bioinformatics 28, 2184-2185 (2012).

32. Subramanian, A. et al. Gene set enrichment analysis: a knowledge-based approach for interpreting genome-wide expression profiles. Proc. Natl Acad. Sci. USA 102, 15545-15550 (2005).

33. Li, Z. et al. ASXL1 interacts with the cohesin complex to maintain chromatid separation and gene expression for normal hematopoiesis. Sci. Adv. 3, e1601602 (2017).

34. Zhang, P. et al. Loss of Asxl1 alters self-renewal and cell fate of bone marrow stromal cell, leading to Bohring-Opitz-like syndrome in mice. Stem Cell Rep. 6, 914-925 (2016).

35. Zang, C. et al. A clustering approach for identification of enriched domains from histone modification ChIP-Seq data. Bioinformatics 25, 1952-1958 (2009).

36. Shao, Z., Zhang, Y., Yuan, G. C., Orkin, S. H. \& Waxman, D. J. MAnorm: a robust model for quantitative comparison of ChIP-Seq data sets. Genome Biol. 13, R16 (2012).

37. Aszodi, A. MULTOVL: fast multiple overlaps of genomic regions Bioinformatics 28, 3318-3319 (2012).

38. Ramirez, F., Dundar, F., Diehl, S., Gruning, B. A. \& Manke, T. deepTools: a flexible platform for exploring deep-sequencing data. Nucleic Acids Res. 42, W187-W191 (2014).

39. Ji, H., Jiang, H., Ma, W., Johnson, D. S., Myers, R. M. \& Wong, W. H. An integrated software system for analyzing ChIP-chip and ChIP-seq data. Nat Biotechnol. 26, 1293-1300 (2008). 
40. Huang, da, W., Sherman, B. T. \& Lempicki, R. A. Systematic and integrative analysis of large gene lists using DAVID bioinformatics resources. Nat. Protoc. 4, 44-57 (2009).

41. Isserlin, R., Merico, D., Voisin, V. \& Bader, G. D. Enrichment map - a Cytoscape app to visualize and explore OMICs pathway enrichment results. F1000Res 3, 141 (2014).

42. Members, B. I. G. D. C. The BIG Data Center: from deposition to integration to translation. Nucleic Acids Res. 45, D18-D24 (2016).

\section{Acknowledgements}

This work was supported by grants from the National Institutes of Health (CA172408 and CA185751 to F.-C.Y. and M.X., and HL112294 to M.X.), the National Key Basic Research Program of China (2014CB542001 to Q.-F.W.), the External Cooperation Program of BIC, Chinese Academy of Sciences (grant 153F11KYSB20150013 to Q.-F.W.), the National Natural Science Foundation of China (81470340 to F.H.) and the Youth Innovation Promotion Association of Chinese Academy of Sciences (F.H.). We thank Zehui Xu, Yuguang Ban, Jin Li and Ehsan Hajiramezanali for the statistical data analysis. We also thank the services provided by the Satellite Histological Core, Flow Cytometry Core, Oncogenomics Core and Biostatistics and Bioinformatics Core Facilities of Sylvester Comprehensive Cancer Center, University of Miami Miller School of Medicine.

\section{Author contributions}

F.-C.Y. conceived the project. J.L., F.H., P.Z., Q.-F.W., M.X. and F.-C.Y. designed the study. J.L., P.Z., S.C., H.S., Y.G., H.Y., N.M., S.G. and Z.L. performed the cellular and molecular experiments. F.H., P.Z., J.L., Y.S., Z.G., S.W., X.C., P.Y., Q.-F.W. and F.-C.Y. performed the RNA-seq and ChIP-seq analysis. J.L., S.C., M.X. and F.-C.Y. reviewed the blood smears and histopathological sections. J.L., P.Z., Y.Z., L.W., M.L., Q.W., S.N., M.X.

and F.-C.Y. discussed and analysed the data. J.L., F.H., P.Z., Q.-F.W., M.X. and F.-C.Y. wrote the manuscript. All authors reviewed and approved the manuscript.

\section{Additional information}

Supplementary Information accompanies this paper at http://www.nature.com/ naturecommunications

Competing interests: The authors declare no competing financial interests.

Reprints and permission information is available online at http://npg.nature.com/ reprintsandpermissions/

How to cite this article: Li, J. et al. Loss of Asxl2 leads to myeloid malignancies in mice. Nat. Commun. 8, 15456 doi: 10.1038/ncomms15456 (2017).

Publisher's note: Springer Nature remains neutral with regard to jurisdictional claims in published maps and institutional affiliations.

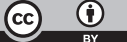
article are included in the article's Creative Commons license, unless indicated otherwise in the credit line; if the material is not included under the Creative Commons license, users will need to obtain permission from the license holder to reproduce the material. To view a copy of this license, visit http://creativecommons.org/licenses/by/4.0/

(C) The Author(s) 2017 